\title{
Explicit flock solutions for Quasi-Morse potentials
}

\author{
J. A. CARRILLO, Y. HUANG and S. MARTIN \\ Department of Mathematics, Imperial College London, London SW7 2AZ, UK \\ email: \{carrillo, yanghong.huang, stephan.martin\}@imperial.ac.uk
}

(Received 13 August 2013; revised 18 March 2014; accepted 19 March 2014;

first published online 15 April 2014)

\begin{abstract}
We consider interacting particle systems and their mean-field limits, which are frequently used to model collective aggregation and are known to demonstrate a rich variety of pattern formations. The interaction is based on a pairwise potential combining short-range repulsion and long-range attraction. We study particular solutions, which are referred to as flocks in the second-order models, for the specific choice of the Quasi-Morse interaction potential. Our main result is a rigorous analysis of continuous, compactly supported flock profiles for the biologically relevant parameter regime. Existence and uniqueness are proven for three space dimensions, while existence is shown for the two-dimensional case. Furthermore, we numerically investigate additional Morse-like interactions to complete the understanding of this class of potentials.
\end{abstract}

Key words: Integral equations; Swarming patterns; Non-local problems; Bessel functions

\section{Introduction}

Self-organization, complex pattern formation, and rich dynamic structures are common features of collective motion of individuals. Fish shoals, bird flocks, insects swarms, myxobacteria formations, and many others are just particular instances of these fascinating phenomena [8,14]. A large number of models have been introduced based on social interaction mechanisms between individuals, namely: long-range attraction, short-range repulsion, and alignment; see [18,21,26], for example.

Here we concentrate on the by-now classical models in which the attraction and repulsion between individuals are taken into account via a pairwise radial potential $W(x)=U(|x|)$. The first-order aggregation model of swarming $([6,19,20,28])$ then reads

$$
\frac{d x_{i}}{d t}=-\frac{1}{N} \sum_{j \neq i} \nabla W\left(x_{i}-x_{j}\right) .
$$

For the second-order model of swarming, an asymptotic cruise speed is fixed by the balance of self-propulsion and friction terms, see $[15,24]$. The governing system of equations for 
the particle dynamics $\left(x_{i}, v_{i}\right) \in \mathbb{R}^{n} \times \mathbb{R}^{n}, i=1,2, \ldots, N$ is

$$
\begin{aligned}
& \frac{d x_{i}}{d t}=v_{i} \\
& \frac{d v_{i}}{d t}=\alpha v_{i}-\beta\left|v_{i}\right|^{2} v_{i}-\frac{1}{N} \sum_{j \neq i} \nabla W\left(x_{i}-x_{j}\right) .
\end{aligned}
$$

The self-propulsion term $\alpha v_{i}-\beta\left|v_{i}\right|^{2} v_{i}$ with the Rayleigh-type dissipation can also be generalized to the form $f\left(\left|v_{i}\right|\right) v_{i}$ for some function $f:[0, \infty) \rightarrow \mathbb{R}$, such that $f(0)>0$ and $f(v)$ becomes negative when $v$ is large enough. In both models, the potential $W$ is assumed to be repulsive at short range $(U(r)$ decreases for small $r>0)$ and attractive at long range $(U(r)$ increases for $r$ large enough). The most popular one used in the literature is the Morse-type potential [15,24]:

$$
U(r)=C_{\mathscr{R}} e^{-r / \ell_{\mathscr{A}}}-C_{\mathscr{A}} e^{-r / \ell_{\mathscr{A}}},
$$

where $C_{\mathscr{R}}, C_{\mathscr{A}}$ specify the strength of the repulsive and attractive forces, and $\ell_{\mathscr{R}}, \ell_{\mathscr{A}}$ specify their length scales.

Depending on the parameters, system (1.2) exhibits a rich variety of patterns: flocks, rotating mills, rings, and clumps [15,24]. To further study the emergence and bifurcation of these patterns, one has to resort to the corresponding continuum equations, derived from either kinetic theory or mean field approximation in the limit when the number of particles $N$ goes to infinity. The system of equations for the continuous density $\rho$ and the velocity $u$ reads $[9,13,24]$

$$
\begin{aligned}
& \frac{\partial \rho}{\partial t}+\operatorname{div}(\rho u)=0 \\
& \frac{\partial u}{\partial t}+(u \cdot \nabla) u=\left(\alpha-\beta|u|^{2}\right) u-\nabla W \star \rho,
\end{aligned}
$$

where $W \star \rho$ is the convolution between $W$ and $\rho$. In particular, a coherent moving flock is a solution such that $u(x, t)=u_{0}, \rho(x, t)=\rho_{F}\left(x-u_{0} t\right)$ for some constant velocity $u_{0}$ with $\left|u_{0}\right|^{2}=\frac{\alpha}{\beta}$, and steady density $\rho_{F}$ satisfying the equation $\nabla W \star \rho_{F}=0$ on the support of $\rho_{F}[1,9,11,12]$. If we deal with densities supported on an open set, the existence of flock solutions for (1.2) is reduced to $W \star \rho=D$, on $\operatorname{supp}[\rho]$ for some constant $D$, where the subscript $F$ for the steady flock solution $\rho_{F}$ is dropped in the rest of the paper for simplicity.

As a matter of fact, flock solutions in this generality coincide with the stationary solutions for the first-order continuum model derived from (1.1), which reads

$$
\frac{\partial \rho}{\partial t}+\operatorname{div}((-\nabla W \star \rho) \rho)=0 .
$$

The existence of some particular explicit stationary solutions where the density is uniformly concentrated on a ring [3,23], for both discrete model (1.1) and continuum case (1.5), has led to a thorough study of their stability and properties in the framework of the first-order models $[2,3,7,23,30,31]$. The stability of the ring flock solutions for the secondorder model (1.2) has been recently tackled in [1]. However, in many instances, as in the 
archetypical Morse potentials, we do observe nicely compactly supported radial flocks in simulations. In the rest of this work, we will concentrate in finding non-concentrated flock profiles for both (1.4) and (1.5).

Definition 1.1 (Flock profile) For a given $W$, a flock profile is defined as a radially symmetric continuous probability density $\rho(r)$, compactly supported on a ball of radius $R_{F}$ satisfying the characteristic equation

$$
W \star \rho=D, \quad \text { on } \operatorname{supp}[\rho]=B\left(0, R_{F}\right) \text { for some constant } D \text {. }
$$

Despite their observation in simulations of (1.2) with a variety of attractive-repulsive potentials, there is nearly no analytical study on the existence and bifurcation of these flocks in the parameter space. The reason lies in the great difficulties in solving the integral equation (1.6) for popular potentials such as (1.3). Multiple solutions may exist (see [24]) by a Newton solver, where the non-physical solutions are shown to be unstable. Other available solutions are in general asymptotic, when the the density is concentrated on a thin annulus [4]. Another fully explicit case corresponds to the Newtonian repulsion with quadratic confinement $W(x)=\frac{|x|^{2}}{2}-\frac{|x|^{2-n}}{2-n}$, for which the solution is the characteristic function of a ball with suitable radius. However, for any other member of the family of potentials

$$
W(x)=\frac{|x|^{a}}{a}-\frac{|x|^{b}}{b}, \quad a>b \geqslant 2-n,
$$

with the convention that $\frac{|x|^{0}}{0}=\log x$, they are no longer explicit, see $[2,16,17]$. Moreover, flock profiles play an important role on the dynamics of (1.2) since they form a stable family of attracting solutions as shown in [10] for general potentials under suitable conditions.

One approach to get explicit solutions of equation (1.6) is to replace $W$ with an analytically more tractable kernel, for instance, the so-called Quasi-Morse potential proposed in [12], instead of (1.3). The great simplification with the Quasi-Morse potential comes from an explicit expression of $\rho$, characterized by only three parameters, which is obtained by solving an ordinary differential equation (ODE) derived from (1.6). The three parameters are found in [12] by a numerical procedure involving the computation of the convolution on the left-hand side of (1.6). The resulting numerical solutions in two and three dimensions agree very well with those approximated from particle simulations. In this paper, we show that this computationally intensive convolution can be evaluated as a few algebraic terms, hence the existence/non-existence of the flock profile in the parameter space can be discussed in detail.

We start in Section 2 by summarizing the properties of the Quasi-Morse potentials and deriving new explicit formulas for the convolution (1.6). Section 3 is devoted to the analysis of existence and uniqueness of flock profiles in a three-dimensional (3D) case with respect to the parameter space of the potential. In Section 4, we perform a similar analysis in two dimensions to identify the existence of flock profiles in parameter space. Due to the simplification of the Bessel functions in three dimensions, the expressions are easier to manage, and the result obtained is more complete in three dimensions. Section 5 
deals with further remarks on the Quasi-Morse potentials and asymptotic cases. Finally, we end this work in Section 6 by investigating similar properties in Morse-like potentials to numerically ascertain how generic is the case of the Quasi-Morse potential.

\section{The Quasi-Morse potential and explicit flock profiles in general dimensions}

For completeness, we first review the basic properties and the explicit solutions proposed in [12]. The new pairwise Quasi-Morse potential $W(x)=U(|x|)$ still assumes the form $U(r)=V(r)-V_{\ell}(r)$, where now $V(r)$ is the fundamental solution of the second-order differential operator $\Delta-k^{2}$ Id (i.e., $\left.\Delta V-k^{2} V=\delta_{0}\right)$ and $V_{\ell}(r)=C V(r / \ell)$ is a rescaled version of $V(r)$ (i.e., $\Delta V_{\ell}-\frac{k^{2}}{\ell^{2}} V_{\ell}=\ell^{n-2} \delta_{0}$ ). For simplicity, here the attraction strength $C_{\mathscr{A}}$ and the length scale $\ell_{\mathscr{A}}$ are normalized to be unity, and then $C=C_{\mathscr{R}}$ and $\ell=\ell_{\mathscr{R}}$.

The biologically relevant cases correspond to the radial potential $U(r)$ possessing a unique global minimum at some positive radius. It was proven in [12] that the biologically relevant parameter region is $C \ell^{n-2}>1$ and $\ell<1$ for dimensions one to three. The explicit expressions for $V(r)$ in these dimensions are given in [12] as $-e^{-k r} / 2 k,-K_{0}(k r) / 2 \pi$, and $-e^{-k r} / 4 \pi r$ respectively. To present the discussion in a unified context for dimension $n$, we write $V(r)$ in terms of the modified Bessel functions of the second kind [25], i.e.,

$$
V(r)=-(2 \pi)^{-\frac{n}{2}} r^{1-\frac{n}{2}} k^{\frac{n}{2}-1} K_{\frac{n}{2}-1}(k r),
$$

and correspondingly

$$
U(r)=(2 \pi)^{-\frac{n}{2}} r^{1-\frac{n}{2}} k^{\frac{n}{2}-1}\left(C \ell^{\frac{n}{2}-1} K_{\frac{n}{2}-1}(k r / \ell)-K_{\frac{n}{2}-1}(k r)\right) .
$$

In particular, $U$ reduces to the conventional Morse potential (1.3) in dimension one as $K_{-\frac{1}{2}}(x)=\sqrt{\frac{\pi}{2 x}} e^{-x}$ (see Appendix A, with other properties of the Bessel function $J_{v}(x)$ and the modified Bessel functions $K_{v}(x)$ and $I_{v}(x)$ used later).

One of the advantages of the Quasi-Morse potential (2.1) is that the integral equation (1.6) can be transformed into a second-order ODE for the radial density $\rho(r)$. Applying the operators $\Delta-k^{2}$ Id and $\Delta-\frac{k^{2}}{\ell^{2}}$ Id to both sides of $(1.6)$ as in $[4,12]$, the density $\rho$ now satisfies

$$
\Delta \rho+A \rho=\frac{k^{4}}{\ell^{2}-C \ell^{n}} D, \quad \text { on } \operatorname{supp} \rho,
$$

with the aggregate potential parameter $A=k^{2}\left(1-C \ell^{n}\right) /\left(C \ell^{n}-\ell^{2}\right)$. In radial coordinate $r$, this equation reads

$$
\frac{1}{r^{n-1}} \frac{d}{d r} r^{n-1} \frac{d \rho}{d r} \pm a^{2} \rho=\frac{k^{4}}{\ell^{2}-C \ell^{n}} D, \quad a=\sqrt{|A|} .
$$

The general solution, assumed to be bounded at the origin, takes the form (see [12] for $n=2,3$ )

$$
\rho(r)=\left\{\begin{aligned}
\mu_{1} r^{1-\frac{n}{2}} J_{\frac{n}{2}-1}(a r)+\mu_{2}, & & A>0, \\
\mu_{1} r^{2}+\mu_{2}, & A & =0, \\
\mu_{1} r^{1-\frac{n}{2}} I_{\frac{n}{2}-1}(a r)+\mu_{2}, & & A<0,
\end{aligned}\right.
$$


on $[0, R]$ and $\rho(r) \equiv 0$ on $(R, \infty)$. For any fixed radius $R$, parameters $\mu_{1}$ and $\mu_{2}$ have to be adjusted to fit the integral equation (1.6) and ensure positivity of $\rho(r)$ on $r \in[0, R]$. In fact, this is exactly how the numerical solutions are obtained in [12], where the observed flock profiles exist only when $A>0$. Despite the perfect agreement with particle simulations, the convolution $W \star \rho$ remains the bottleneck of the computation. In this paper, we show that the convolution can also be reduced to a few algebraic terms, eventually leading to the rigorous existence/non-existence proofs of radial solutions in different parameter regimes.

The simplification of the convolution $W \star \rho$ is suggested by the following observation: When operators $\Delta-k^{2} \mathrm{Id}$ and $\Delta-\frac{k^{2}}{\ell^{2}} \mathrm{Id}$ are applied on both sides of (1.6), we get a fourth-order ODE (in the radial coordinate $r$ ),

$$
\left(\frac{1}{r^{n-1}} \frac{d}{d r} r^{n-1} \frac{d}{d r}-\frac{k^{2}}{\ell^{2}}\right)\left(\frac{1}{r^{n-1}} \frac{d}{d r} r^{n-1} \frac{d}{d r}-k^{2}\right) W \star \rho=\frac{k^{4}}{\ell^{2}} D
$$

for the radial function $W \star \rho$, which is equivalent to (2.2). The general solution of the fourth-order ODE takes the form

$$
\begin{aligned}
(W \star \rho)(r)=D & +\lambda_{1} r^{1-\frac{n}{2}} I_{\frac{n}{2}-1}(k r / \ell)+\lambda_{2} r^{1-\frac{n}{2}} I_{\frac{n}{2}-1}(k r) \\
& +\lambda_{3} r^{1-\frac{n}{2}} K_{\frac{n}{2}-1}(k r / \ell)+\lambda_{4} r^{1-\frac{n}{2}} K_{\frac{n}{2}-1}(k r), \quad 0 \leqslant r \leqslant R,
\end{aligned}
$$

for some coefficients $\lambda_{1}, \ldots, \lambda_{4}$. We will find the desired flock profiles when all $\lambda_{i}$ vanish and thus (1.6) is fulfilled. We first note that $\lambda_{3}$ and $\lambda_{4}$ have to vanish in order to have a bounded solution at the origin with bounded derivatives. Imposing that $\lambda_{1}$ and $\lambda_{2}$ vanish will lead to necessary and sufficient conditions for a flock profile. Following this strategy, $D, \lambda_{1}$, and $\lambda_{2}$ will be expressed in terms of the support size $R$ and the coefficients $\mu_{1}, \mu_{2}$ by inserting (2.3) on the left-hand side of (2.4).

First, we compute $\lambda_{1}, \lambda_{2}$ for the explicit solution in (2.3). It turns out that the convolution $W \star \rho$ can be obtained by direct integrations. To start, because of the radial symmetry, $W \star \rho$ can be written as

$$
(W \star \rho)(x)=\int_{|y| \leqslant R} W(x-y) \rho(|y|) d y=\int_{0}^{R}\left(\int_{\partial B(0,1)} W(x-s \omega) d \omega\right) \rho(s) s^{n-1} d s .
$$

This convolution, as a function of $r=|x|$, simplifies in the particular case of the QuasiMorse potential $W(x)=V(|x|)-C V(|x| / \ell)$. In fact, the integral on the unit sphere $\partial B(0,1)$ above can be evaluated using the following formula (see [27, p. 90]):

$$
\begin{gathered}
\int_{0}^{\pi}\left(a^{2}+b^{2}-2 a b \cos \theta\right)^{-v / 2} K_{v}\left(\left(a^{2}+b^{2}-2 a b \cos \theta\right)^{1 / 2}\right) \sin ^{2 v} \theta d \theta \\
=\pi^{1 / 2} \Gamma\left(\frac{1}{2}+v\right)\left(\frac{2}{a b}\right)^{v} I_{v}(\min (a, b)) K_{v}(\max (a, b)) .
\end{gathered}
$$

Let us detail the computation of this angular integral for the second component $V_{\ell}(r)=$ $C V(r / \ell)$ of $W$, as the integral for $V(r)$ is the special case of $C=\ell=1$. Setting $v=n / 2-1$, 
$a=k r / \ell$, and $b=k s / \ell$, the angular integration involving $V_{\ell}$ in (2.5) reads

$$
\begin{aligned}
\int_{\partial B(0,1)} V_{\ell}(x-s \omega) d \omega & =-C \frac{2 \pi^{\frac{n-1}{2}}}{\Gamma\left(\frac{n-1}{2}\right)}(2 \pi)^{-\frac{n}{2}} k^{n-2} \int_{0}^{\pi} D(\theta)^{-v / 2} K_{v}\left(D(\theta)^{1 / 2}\right) \sin ^{2 v} \theta d \theta \\
& =-C \ell^{n-2}(r s)^{1-\frac{n}{2}} I_{\frac{n}{2}-1}\left(\frac{k}{\ell} \min (r, s)\right) K_{\frac{n}{2}-1}\left(\frac{k}{\ell} \max (r, s)\right)
\end{aligned}
$$

where $D(\theta)=\frac{k^{2}}{\ell^{2}}\left(r^{2}+s^{2}-2 r s \cos \theta\right)$. As a result, the convolution (2.5) becomes an integral in $s$ only and the convolution of the repulsive potential $V_{\ell}$ with a density $\rho$ supported on the ball $B(0, R)$ is

$$
\begin{aligned}
V_{\ell} \star \rho(x)= & C \ell^{n-2} r^{1-\frac{n}{2}}\left[K_{\frac{n}{2}-1}(k r / \ell) \int_{0}^{r} s^{\frac{n}{2}} I_{\frac{n}{2}-1}(k s / \ell) \rho(s) d s\right. \\
& \left.+I_{\frac{n}{2}-1}(k r / \ell) \int_{r}^{R} S^{\frac{n}{2}} K_{\frac{n}{2}-1}(k s / \ell) \rho(s) d s\right]
\end{aligned}
$$

for $0 \leqslant r=|x| \leqslant R$. This integral, when $\rho$ takes the special form (2.3), can be further simplified using various integral identities of (modified) Bessel functions. Since these algebraic manipulations do not bring any further insights, we postponed these to Appendix B. The final result, whose general forms are already expected from (2.4), is as follows.

Proposition 2.1 Given the Quasi-Morse potential $W(x)=U(|x|)$ in (2.1) and $\rho$ defined in (2.3), the convolution $W \star \rho$ has the expression:

$$
W \star \rho(x)=\left\{\begin{aligned}
\frac{\mu_{2}}{k^{2}}\left(C \ell^{n}-1\right)+\frac{R^{\frac{n}{2}}}{k} r^{1-\frac{n}{2}}\left[B_{+}(1) K_{\frac{n}{2}}(k R) I_{\frac{n}{2}-1}(k r)\right. & \\
\left.-C \ell^{n-1} B_{+}(\ell) K_{\frac{n}{2}}(k R / \ell) I_{\frac{n}{2}-1}(k r / \ell)\right] & A>0, \\
\frac{2 n \mu_{1}}{k^{4}}\left(C \ell^{n+2}-1\right)+R^{\frac{n}{2}} r^{1-\frac{n}{2}}\left[B_{0}(1) K_{\frac{n}{2}}(k R) I_{\frac{n}{2}-1}(k r)\right. & \\
\left.-C \ell^{n-1} B_{0}(\ell) K_{\frac{n}{2}}(k R / \ell) I_{\frac{n}{2}-1}(k r / \ell)\right] & A=0, \\
\frac{\mu_{2}}{k^{2}}\left(C \ell^{n}-1\right)+\frac{R^{\frac{n}{2}}}{k} r^{1-\frac{n}{2}}\left[B_{-}(1) K_{\frac{n}{2}}(k R) I_{\frac{n}{2}-1}(k r)\right. & \\
\left.-C \ell^{n-1} B_{-}(\ell) K_{\frac{n}{2}}(k R / \ell) I_{\frac{n}{2}-1}(k r / \ell)\right] & A<0,
\end{aligned}\right.
$$

where $B_{+}(\xi)=\tilde{B}_{+}(\xi) \mu_{1}+\mu_{2}, B_{0}(\xi)=\tilde{B}_{0}(\xi) \mu_{1}+\mu_{2}, B_{-}(\xi)=\tilde{B}_{-}(\xi) \mu_{1}+\mu_{2}$, and

$$
\begin{aligned}
& \tilde{B}_{+}(\xi)=R^{1-\frac{n}{2}}\left(1+\frac{a^{2} \xi^{2}}{k^{2}}\right)^{-1}\left[J_{\frac{n}{2}-1}(a R) \frac{K_{\frac{n}{2}-2}(k R / \xi)}{K_{\frac{n}{2}}(k R / \xi)}+\frac{a \xi}{k} J_{\frac{n}{2}-2}(a R) \frac{K_{\frac{n}{2}-1}(k R / \xi)}{K_{\frac{n}{2}}(k R / \xi)}\right], \\
& \tilde{B}_{0}(\xi)=\frac{2 \xi}{k} R \frac{K_{\frac{n}{2}+1}(k R / \xi)}{K_{\frac{n}{2}}(k R / \xi)}+1 \\
& \tilde{B}_{-}(\xi)=R^{1-\frac{n}{2}}\left(1-\frac{a^{2} \xi^{2}}{k^{2}}\right)^{-1}\left[I_{\frac{n}{2}-1}(a R) \frac{K_{\frac{n}{2}-2}(k R / \xi)}{K_{\frac{n}{2}}(k R / \xi)}+\frac{a \xi}{k} I_{\frac{n}{2}-2}(a R) \frac{K_{\frac{n}{2}-1}(k R / \xi)}{K_{\frac{n}{2}}(k R / \xi)}\right] .
\end{aligned}
$$


Table 1. Formulas for $\lambda_{1}$ and $\lambda_{2}$ in (2.4) when $\rho$ is given by (2.3)

\begin{tabular}{ccc}
\hline \hline & $\lambda_{1}$ & $\lambda_{2}$ \\
\hline$A>0$ & $-C \frac{R^{\frac{n}{2}}}{k} \ell^{n-1} B_{+}(\ell) K_{\frac{n}{2}}(k R / \ell)$ & $\frac{R^{\frac{n}{2}}}{k} \ell^{n-1} B_{+}(1) K_{\frac{n}{2}}(k R)$ \\
$A=0$ & $-C R^{\frac{n}{2}} \ell^{n-1} B_{0}(\ell) K_{\frac{n}{2}}(k R / \ell)$ & $R^{\frac{n}{2}} \ell^{n-1} B_{0}(1) K_{\frac{n}{2}}(k R)$ \\
$A<0$ & $-C \frac{R^{\frac{n}{2}}}{k} \ell^{n-1} B_{-}(\ell) K_{\frac{n}{2}}(k R / \ell)$ & $\frac{R^{\frac{n}{2}}}{k} \ell^{n-1} B_{-}(1) K_{\frac{n}{2}}(k R)$ \\
\hline
\end{tabular}

From now on, subscripts of $B$ or $\tilde{B}$, which indicate the sign of $A$, will be omitted when the discussion is relevant to all three cases (similarly for other variables such as the coefficient matrix $M$ mentioned below).

Equipped with these expressions of the convolution, we further study the existence/nonexistence of the flock profile in parameter space. As mentioned above, the explicit formulas allow us to write $\lambda_{1}$ and $\lambda_{2}$, by plugging (2.9) into (2.4), in terms of $\mu_{1}, \mu_{2}$, and $R$. Since $r^{1-n / 2} I_{\frac{n}{2}-1}(k r)$ and $r^{1-n / 2} I_{\frac{n}{2}-1}(k r / \ell)$ are independent, we deduce the formulas in Table 1.

For the flock profile we are interested in, $\lambda_{1}$ and $\lambda_{2}$ must be zero. In view of Table 1 , this is equivalent to the conditions $B(\ell)=0, B(1)=0$, since $K_{v}(x)$ is non-zero on $(0, \infty)$. Therefore, there exists a flock profile only if the homogeneous equations for $\boldsymbol{\mu}=\left(\mu_{1}, \mu_{2}\right)^{T}$

$$
M \boldsymbol{\mu}=\left(\begin{array}{ll}
\tilde{B}(\ell) & 1 \\
\tilde{B}(1) & 1
\end{array}\right)\left(\begin{array}{l}
\mu_{1} \\
\mu_{2}
\end{array}\right)=\left(\begin{array}{l}
0 \\
0
\end{array}\right)
$$

are satisfied. These two homogeneous equations, together with the total unit mass constraint for the non-negative density $\rho$, determine the three characterizing parameters $\left(\mu_{1}, \mu_{2}, R_{F}\right)$ of flock profile.

A careful examination of the three equations shows that the radius of the support $R$ is determined by the scalar equation $\operatorname{det} M=\tilde{B}(\ell)-\tilde{B}(1)=0$, since $\mu_{1}$ and $\mu_{2}$ must be nontrivial solutions of (2.11). In fact, all the subsequent results are based on studying the roots of det $M$ and the properties of $\tilde{B}(\xi)$ as functions of $R$. Below we focus on the physical $2 \mathrm{D}$ and $3 \mathrm{D}$ cases, on the biologically relevant regime $\ell<1, C \ell^{n-2}>1$. However, unlike the unified derivation of the convolution to (2.9), the existence/non-existence question is much more complicated and has to be treated separately.

The main results of this paper (Theorems 3.1 and 4.1) in the biologically relevant regimes are summarized in Figure 1. We show the existence and uniqueness of flock profiles in the $3 \mathrm{D}$ case for $A>0$ and non-existence otherwise. In the $2 \mathrm{D}$ case, we show the existence of flock profiles for $A>0$ and non-existence otherwise. However, we cannot conclude the uniqueness of flock profiles. Because of the connection of the (modified) Bessel functions in three dimension (and odd dimensions in general) with the well-known trigonometric functions, we consider this case first.

\section{Existence theory of flock profiles in three dimensions}

We first turn to the existence theory of flock profiles in three space dimensions, as in this case the Bessel functions in the potential as well as in all subsequent computations reduce to trigonometric functions (see Appendix A). The aggregate potential parameter 


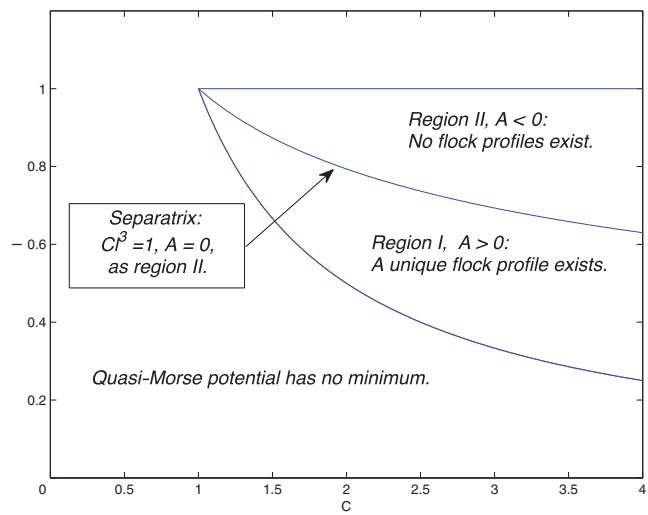

(a) Results of Section $3, n=3$

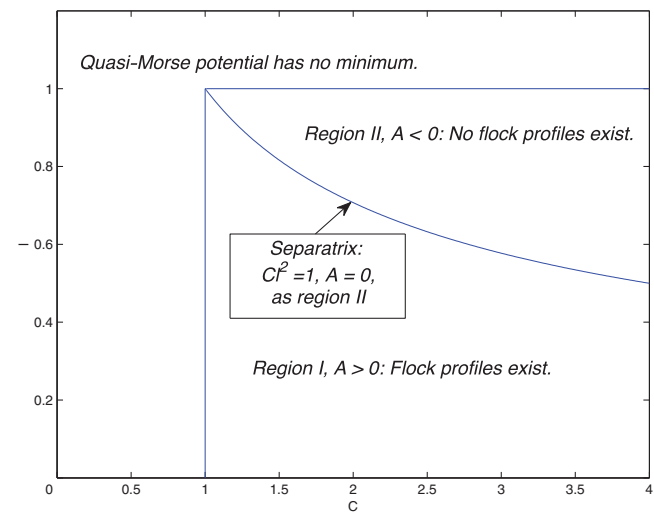

(b) Results of Section $4, n=2$

FIGURE 1. (Colour online) Phase-diagrams of parameters $C, \ell$ for the Quasi-Morse potential illustrating the combined results of Theorems 3.1 and 4.1. For both dimensions $n=2,3$, the aggregate parameter $A$ divides the biologically relevant parameter space $\left\{(C, \ell) \mid C \ell^{n-2}>1, \ell<1\right\}$ into two subregions I and II by the curve $C \ell^{n}=1$. In region I, $A>0$, a flock profile always exists. In region II and the separatrix, $A \leqslant 0$, no flock profiles exist. When $n=3$, existing flock profiles are additionally known to be unique.

$A$ is computed as

$$
A=k^{2}\left(1-C \ell^{3}\right) /\left(C \ell^{3}-\ell^{2}\right),
$$

and the expressions (2.10) used in the explicit convolution (2.9) simplify to

$$
\begin{gathered}
\tilde{B}_{+}(\xi)=\sqrt{\frac{2}{a \pi}}\left(1+\frac{a^{2} \xi^{2}}{k^{2}}\right)^{-1}\left[\sin a R+\frac{a \xi}{k} \cos a R\right] \frac{k}{k R+\xi}, \\
\tilde{B}_{0}(\xi)=\frac{2 \xi}{k^{2}} \frac{(k R)^{2}+3 k R \xi+3 \xi^{2}}{k R+\xi}+1, \\
\tilde{B}_{-}(\xi)=\sqrt{\frac{2}{a \pi}}\left(1-\frac{a^{2} \xi^{2}}{k^{2}}\right)^{-1}\left[\sinh a R+\frac{a \xi}{k} \cosh a R\right] \frac{k}{k R+\xi},
\end{gathered}
$$

as $K_{3 / 2}(x) / K_{1 / 2}(x)=1+1 / x$ and $K_{5 / 2}(x) / K_{3 / 2}(x)=\left(x^{2}+3 x+3\right) / x(x+1)$. Based on numerical findings, it has been conjectured in [12] that flock profiles can be found only for the Quasi-Morse potentials where $A>0$. The insight from the above explicit calculations enables us now to prove existence and uniqueness of flock profiles, and thus to analytically investigate the phase diagram of parameters $C, \ell$ in the biologically relevant scenarios $C \ell>1, \ell<1$ (see Figure 1). In fact, the following theorem holds.

Theorem 3.1 Let $W$ be a Quasi-Morse potential in space dimension $n=3$ with parameters within the biologically relevant regime $C \ell>1, \ell<1$. Then flock profiles exist if and only if $A>0$. Furthermore, if $A>0$, there exists a unique flock profile.

To prove Theorem 3.1, we begin with the discussion on the non-existence of flock profiles for $A \leqslant 0$. 
Proof (Theorem 3.1, non-existence for $A \leqslant 0$ ) When $A=0$, for all $R$, we can show $\operatorname{det} M_{0}=\tilde{B}_{0}(\ell)-\tilde{B}_{0}(1)<0$ by a straightforward explicit computation using $(3.2 \mathrm{~b})$. We skip that calculation here as the case $A=0$ will also be proven in general dimensions in Theorem 4.1.

Next, suppose that $A<0$. From (3.1), this implies $C \ell^{3}>\ell$ as $C \ell>1, \ell<1$ and furthermore, we have $a^{2}=-A=k^{2}\left(1-C \ell^{3}\right) /\left(\ell^{2}-C \ell^{3}\right)$. The determinant of $M_{-}$ simplifies to

$$
\begin{aligned}
\operatorname{det} M_{-}= & \tilde{B}_{-}(\ell)-\tilde{B}_{-}(1)=\sqrt{\frac{2}{\pi a R^{2}} \frac{\ell^{2}(C \ell-1)}{1-\ell^{2}}} . \\
& {\left[\left(\frac{1}{C \ell^{3}} \frac{k R}{k R+\ell}-\frac{k R}{k R+1}\right) \sinh a R+\frac{a}{k}\left(\frac{1}{C \ell^{2}} \frac{k R}{k R+\ell}-\frac{k R}{k R+1}\right) \cosh a R\right] } \\
= & \sqrt{\frac{2}{\pi a}} \frac{k \ell^{2}(C \ell-1)}{1-\ell^{2}} \frac{\cosh a R}{C \ell^{3}(k R+\ell)(k R+1)} f_{-}(R),
\end{aligned}
$$

where

$$
f_{-}(R)=\frac{a \ell}{k}\left(1-C \ell^{3}\right)+k R\left(1-C \ell^{3}\right) \tanh a R+\left(\ell-C \ell^{3}\right) a R+\left(1-C \ell^{4}\right) \tanh a R .
$$

Clearly, the sign of det $M_{-}$is determined by the sign of $f_{-}(R)$. The first two terms in (3.3) are negative. If $C \ell^{4}>1$, the last two terms are negative as $C \ell^{3}>1 \Rightarrow C \ell^{3}>\ell$. If to the contrary $C \ell^{4} \leqslant 1$, the sum of the last two terms in (3.3) satisfies

$$
\left(\ell-C \ell^{3}\right) a R+\left(1-C \ell^{4}\right) \tanh a R<\left(1+\ell-C \ell^{3}-C \ell^{4}\right) a R=(1+\ell)\left(1-C \ell^{3}\right) a R<0,
$$

as $\tanh a R \leqslant a R$. Thus, $\operatorname{det} M_{-}<0$ for all $R>0$ and there is no real positive root of $\operatorname{det} M_{-}$.

Proving existence of a unique flock profile when $A>0$ is more difficult and relies on various properties of the trigonometric representation of the original half-integer order Bessel functions. Our goal is to show that $\operatorname{det} M_{+}$is oscillatory with decaying amplitude, implying the existence of infinitely many positive roots $R_{j}^{*}, j=1,2, \ldots$, for $\operatorname{det} M_{+}=0$. However, only the first positive root gives rise to a strictly positive density on the support $\left[0, R_{1}^{*}\right]$, and the density for any other roots must be negative somewhere on the support $\left[0, R_{j}^{*}\right], j \geqslant 2$. This asserted behaviour of $\operatorname{det} M_{+}$for $R>0$ is illustrated in Figure 2, with particular parameters taken from [12].

Proof (Theorem 3.1, existence and uniqueness for $A>0$ ) The proof is separated into several steps.

1. There are infinitely many positive roots for $\operatorname{det} M_{+}=0$. From (3.2a), the determinant $\operatorname{det} M_{+}=\tilde{B}_{+}(\ell)-\tilde{B}_{+}(1)$ can be written as

$$
\begin{aligned}
\operatorname{det} M_{+}= & k \sqrt{\frac{2}{a \pi}}\left(\frac{1}{\left(1+a^{2} \ell^{2} / k^{2}\right)(k R+\ell)}-\frac{1}{\left(1+a^{2} / k^{2}\right)(k R+1)}\right) \sin a R \\
& +\sqrt{\frac{2 a}{\pi}}\left(\frac{\ell}{\left(1+a^{2} \ell^{2} / k^{2}\right)(k R+\ell)}-\frac{1}{\left(1+a^{2} / k^{2}\right)(k R+1)}\right) \cos a R .
\end{aligned}
$$



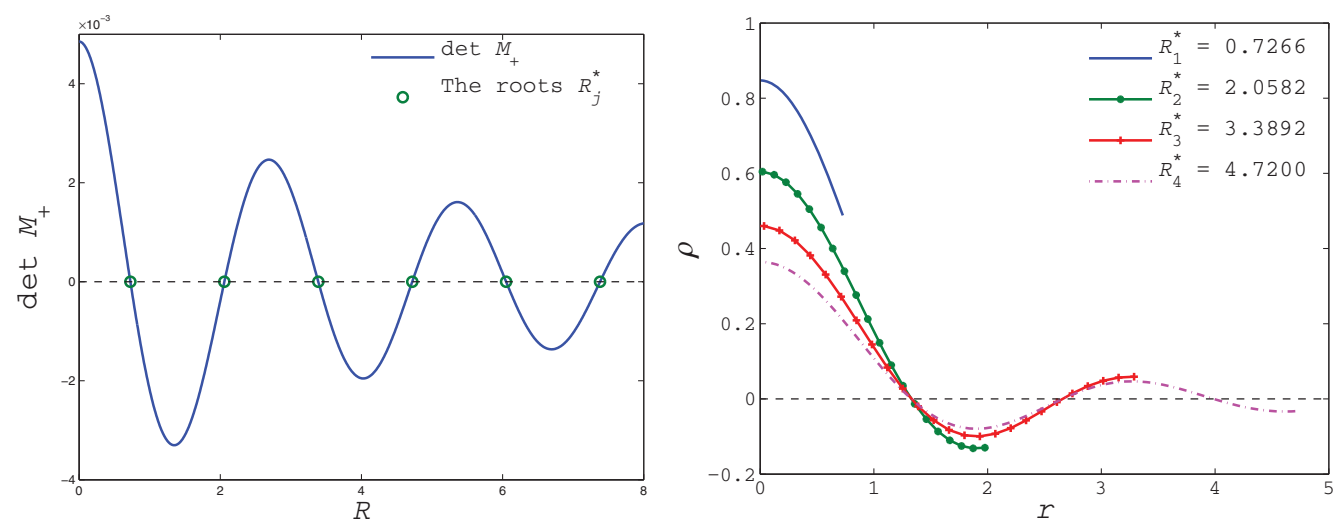

FIGURE 2. (Colour online) Multiple zeros $R^{*}$ of the equation $\operatorname{det} M_{+}=0$ (left) and the corresponding densities (right). Only the first zero $R_{1}^{*}$ gives rise to strict positive density $\rho(r)$ on the support. Here the parameters, $C=1.255, \ell=0.8, k=0.2, A=5.585$ (or $a=2.362$ ), are the same as in [12].

We observe that the coefficient of $\sin a R$ in the above expression is positive, since $(1+$ $\left.a^{2} \ell^{2} / k^{2}\right)^{-1}>\left(1+a^{2} / k^{2}\right)^{-1}$ and $(k R+\ell)^{-1}>(k R+1)^{-1}$. Evaluating $\operatorname{det} M_{+}$at $\tilde{R}_{j}=$ $(j-1 / 2) \pi / a, j=1,2, \ldots$, the roots of $\cos a R$, we deduce that

$$
\left.\operatorname{det} M_{+}\right|_{R=\tilde{R}_{j}}=(-1)^{j} k \sqrt{\frac{2}{a \pi}}\left(\frac{1}{\left(1+a^{2} \ell^{2} / k^{2}\right)\left(k \tilde{R}_{j}+\ell\right)}-\frac{1}{\left(1+a^{2} / k^{2}\right)\left(k \tilde{R}_{j}+1\right)}\right)
$$

has alternating signs. Therefore, there is at least one root between $\left(\tilde{R}_{j}, \tilde{R}_{j+1}\right)$, proving the existence of infinitely many positive roots for $\operatorname{det} M_{+}=0$.

2. The function det $M_{+}$has no root on $\left(0, \tilde{R}_{1}\right)$ and has a unique root $R_{j}^{*}$ on $\left(\tilde{R}_{j}, \tilde{R}_{j+1}\right)$, $j=1,2, \ldots$. We write $\operatorname{det} M_{+}$in the following form,

$$
\frac{\operatorname{det} M_{+}}{\cos a R}=k \sqrt{\frac{2}{a \pi}}\left(\frac{1}{\left(1+a^{2} \ell^{2} / k^{2}\right)(k R+\ell)}-\frac{1}{\left(1+a^{2} / k^{2}\right)(k R+1)}\right)(\tan a R+g(R)),
$$

where

$$
\begin{aligned}
g(R) & =\frac{a}{k} \frac{\left(a^{2} \ell-k^{2}\right) k R+a^{2} \ell(\ell+1)}{a^{2}(\ell+1) k R+k^{2}+a^{2}\left(\ell^{2}+\ell+1\right)} \\
& =\frac{a}{k}\left[\frac{a^{2} \ell-k^{2}}{a^{2}(\ell+1)}+\frac{\left(k^{2}+a^{2}\right)\left(k^{2}+a^{2} \ell^{2}\right)}{a^{2}(\ell+1)\left(a^{2}(\ell+1) k R+k^{2}+a^{2}\left(\ell^{2}+\ell+1\right)\right)}\right] .
\end{aligned}
$$

It is easy to check that the roots of $\operatorname{det} M_{+}=0$ are the same as the roots of $\tan a R+g(R)=$ 0 , and this auxiliary function $g$ is used to show various estimates at various stages of the proof below. Note now that function $\tan a R+g(R)$ is strictly increasing on $\left(\tilde{R}_{j}, \tilde{R}_{j+1}\right)$, 


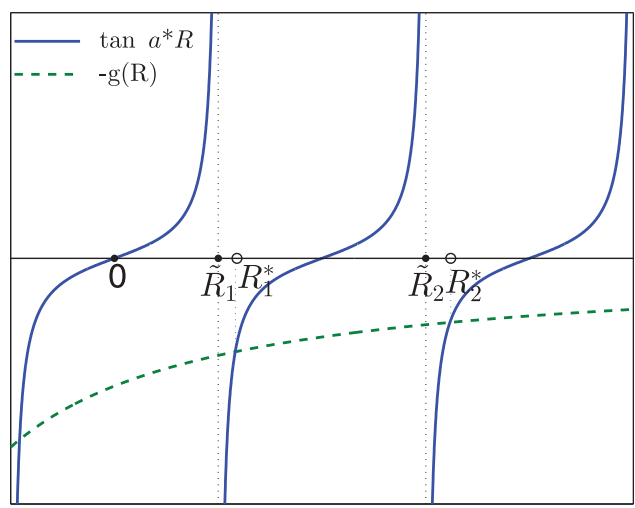

(a) Intersection of $\tan a R$ with $-g(R)$

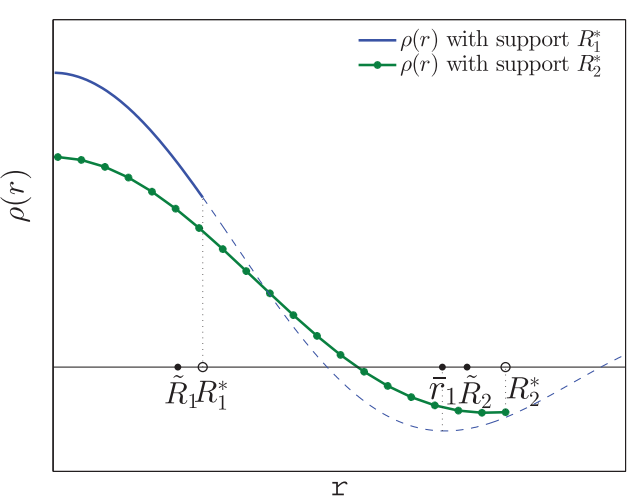

(b) Densities corresponding to $R_{1}^{*}$ and $R_{2}^{*}$

FIGURE 3. (Colour online) Illustrations of the generic properties proved in three dimensions when $A>0$ : (a) $\tan a R$ and $g(R)$ intersects only once at $R_{j}^{*}$ in the interval $\left[\tilde{R}_{j}, \tilde{R}_{j+1}\right)$; (b) Density $\rho(r)$ with support $R_{j}^{*}, j \geqslant 2$ has opposite signs at the origin and at $\tilde{R}_{2}$, while that with $R_{1}^{*}$ is monotonically decreasing from the origin.

since $\frac{d}{d R} \tan a R \geqslant a$ and

$$
g^{\prime}(R)>g^{\prime}(0)=-a \frac{\left(k^{2}+a^{2}\right)\left(k^{2}+a^{2} \ell^{2}\right)}{\left(k^{2}+a^{2}\left(\ell^{2}+\ell+1\right)\right)^{2}}>-a .
$$

Combining this with the fact that

$$
\lim _{R \rightarrow \tilde{R}_{j}^{+}}(\tan a R+g(R))= \pm \infty
$$

we obtain that there is a unique root $R_{j}^{*}$ on $\left(\tilde{R}_{j}, \tilde{R}_{j+1}\right)$, as illustrated in Figure $3($ a). There is no positive root on $\left(0, \tilde{R}_{1}\right)$ because det $M_{+}$is an increasing function on $\left(0, \tilde{R}_{1}\right)$ and

$$
\left.\operatorname{det} M_{+}\right|_{R=0}=\sqrt{\frac{2 a}{\pi}}\left(\left(1+a^{2} \ell^{2} / k^{2}\right)^{-1}-\left(1+a^{2} / k^{2}\right)^{-1}\right)>0 .
$$

3. If $j \geqslant 2$, then the density corresponding to the root $R_{j}^{*}$ cannot be positive at both origin and $\tilde{R}_{2}$. Let $\boldsymbol{\mu}=\left(\mu_{1}, \mu_{2}\right)^{T}$ be the (nontrivial) solution of $\left.M_{+}\right|_{R=R_{j}^{*}} \boldsymbol{\mu}=0$, then the corresponding density is given by

$$
\rho(r)=\mu_{1} r^{-1 / 2} J_{1 / 2}(a r)+\mu_{2}=\mu_{1}\left(\sqrt{\frac{2}{a \pi}} \frac{\sin a r}{r}-\left.\tilde{B}_{+}(1)\right|_{R=R_{j}^{*}}\right) .
$$

A direct evaluation of $\rho$ leads to

$$
\rho(0) \rho\left(\tilde{R}_{2}\right)=\left(\sqrt{\frac{2 a}{\pi}}-\left.\tilde{B}_{+}(1)\right|_{R=R_{j}^{*}}\right)\left(-\sqrt{\frac{8 a}{9 \pi^{3}}}-\left.\tilde{B}_{+}(1)\right|_{R=R_{j}^{*}}\right) \mu_{1}^{2} .
$$


Using (3.2a) and the inequality $\left|\sin a R+\frac{a \xi}{k} \cos a R\right| \leqslant\left(1+\frac{a^{2} \xi^{2}}{k^{2}}\right)^{1 / 2}$, we get

$$
\begin{aligned}
\left|\tilde{B}_{+}(\xi)\right| & =\left|\sqrt{\frac{2}{a \pi}}\left(1+\frac{a^{2} \xi^{2}}{k^{2}}\right)^{-1}\left[\sin a R+\frac{a \xi}{k} \cos a R\right] \frac{k}{k R+\xi}\right| \\
& \leqslant \sqrt{\frac{2}{a \pi}}\left(1+\frac{a^{2} \xi^{2}}{k^{2}}\right)^{-1 / 2} \frac{k}{k R+\xi} .
\end{aligned}
$$

Therefore, since $R_{j}^{*}>\tilde{R}_{2}$,

$$
\left|\tilde{B}_{+}(1)\right|_{R=R_{j}^{*}} \mid \leqslant \sqrt{\frac{2}{a \pi}}\left(1+\frac{a^{2}}{k^{2}}\right)^{-1 / 2} \frac{k}{k R_{j}^{*}+1}<\sqrt{\frac{2}{a \pi}} \frac{1}{\tilde{R}_{2}}=\sqrt{\frac{8 a}{9 \pi^{3}}}<\sqrt{\frac{2 a}{\pi}} .
$$

These estimates imply that $\rho(0) \rho\left(\tilde{R}_{2}\right)<0$, while the physical density $\rho$ must be nonnegative on the support.

4. The density $\rho(r)$ corresponding to the root $R_{1}^{*}$ is decreasing and strictly positive on its support $\left[0, R_{1}^{*}\right]$. Let us first show that $\left.\tilde{B}_{+}(\ell)\right|_{R=R_{1}^{*}}=\left.\tilde{B}_{+}(1)\right|_{R=R_{1}^{*}}<0$. Assume that this is not the case, then $\left.\tilde{B}_{+}(\ell)\right|_{R=R_{1}^{*}}=\left.\tilde{B}_{+}(1)\right|_{R=R_{1}^{*}} \geqslant 0$. Since $\cos a R<0$ for $R \in\left(\tilde{R}_{1}, \tilde{R}_{2}\right)$,

$$
\sin a R_{1}^{*}+\frac{a \ell}{k} \cos a R_{1}^{*}>\sin a R_{1}^{*}+\frac{a}{k} \cos a R_{1}^{*} \geqslant 0
$$

This, together with $\left(1+a^{2} \ell^{2} / k^{2}\right)^{-1}>\left(1+a^{2} / k^{2}\right)^{-1}$ and $\left(k R_{1}^{*}+\ell\right)^{-1}>\left(k R_{1}^{*}+1\right)^{-1}$, implies that $\left.\tilde{B}_{+}(\ell)\right|_{R=R_{1}^{*}}>\left.\tilde{B}_{+}(1)\right|_{R=R_{1}^{*}} \geqslant 0$, leading to a contradiction. Therefore, combining $\left.\tilde{B}_{+}(1)\right|_{R=R_{1}^{*}}<0$ with the fact that $\mu_{2}=-\left.\tilde{B}_{+}(1)\right|_{R=R_{1}^{*}} \mu_{1}$ and $\rho(0)=\sqrt{\frac{2 a}{\pi}} \mu_{1}+\mu_{2}>0$, both $\mu_{1}$ and $\mu_{2}$ must be positive.

It is easy to check that $r^{-1 / 2} J_{1 / 2}(a r)=\sqrt{\frac{2}{a \pi}} \frac{\sin a r}{r}$ is a decreasing function till its first local minimum $\bar{r}_{1}$, determined by

$$
0=\left.\frac{d}{d r} r^{-1 / 2} J_{1 / 2}(a r)\right|_{r=\bar{r}_{1}}=\left.\sqrt{\frac{2}{a \pi}} \frac{\operatorname{arcos} a r-\sin a r}{r^{2}}\right|_{r=\bar{r}_{1}}
$$

or equivalently $a \bar{r}_{1}=\tan a \bar{r}_{1}>0$ with $\bar{r}_{1} \approx 4.49 / a \in\left(\tilde{R}_{1}, \tilde{R}_{2}\right)$. Using the definition (3.5a) of $g$,

$$
\tan a \bar{r}_{1}+g\left(\bar{r}_{1}\right)=a \bar{r}_{1}+g\left(\bar{r}_{1}\right)=\frac{a^{3}}{k} \frac{(\ell+1)\left(1+k \bar{r}_{1}\right)\left(\ell+k \bar{r}_{1}\right)}{a^{2}(\ell+1) k \bar{r}_{1}+k^{2}+a^{2}\left(\ell^{2}+\ell+1\right)}>0 .
$$

Since $R_{1}^{*}$ is the unique root of the strictly increasing function $\tan a R+g(R)$ on the interval $\left(\tilde{R}_{1}, \tilde{R}_{2}\right)$, the fact that $\tan a \bar{r}_{1}+g\left(\bar{r}_{1}\right)>0$ implies that $\bar{r}_{1}>R_{1}^{*}$. Therefore, the density $\rho(r)$ is a decreasing function on $\left[0, R_{1}^{*}\right]$, as illustrated in Figure 3(b). Finally, evaluating $\rho(r)$ at 
the boundary $R=R_{1}^{*}$, we get

$$
\begin{aligned}
\rho\left(R_{1}^{*}\right) & =\mu_{1}\left(\sqrt{\frac{2}{a \pi}} \frac{\sin a R^{*}}{R_{1}^{*}}-\left.\tilde{B}_{+}(1)\right|_{R=R_{1}^{*}}\right) \\
& =-\mu_{1} \sqrt{\frac{2}{a \pi}}\left(1+\frac{a^{2}}{k^{2}}\right)^{-1}\left[\frac{a}{k R_{1}^{*}+1}+\left(\frac{k}{k R_{1}^{*}+1}-\frac{1}{R_{1}^{*}}\left(1+\frac{a^{2}}{k^{2}}\right)\right) \tan a R_{1}^{*}\right] \cos a R_{1}^{*} \\
& =-\mu_{1} \sqrt{\frac{2}{a \pi}}\left(1+\frac{a^{2}}{k^{2}}\right)^{-1}\left[\frac{a}{k R_{1}^{*}+1}-\left(\frac{k}{k R_{1}^{*}+1}-\frac{1}{R_{1}^{*}}\left(1+\frac{a^{2}}{k^{2}}\right)\right) g\left(R_{1}^{*}\right)\right] \cos a R_{1}^{*} \\
& =-\mu_{1} \sqrt{\frac{2 a}{\pi}} \frac{1}{k R_{1}^{*}} \frac{1+\ell+k R_{1}^{*} a^{2}(\ell+1) k R_{1}^{*}+k^{2}+a^{2}\left(\ell^{2}+\ell+1\right)}{\cos a R_{1}^{*}>0 .}
\end{aligned}
$$

This shows that $\rho\left(R_{1}^{*}\right)>0$, and therefore $\rho(r)$ is strictly positive on its support, which completes the proof.

\section{Existence theory of flock profiles in two dimensions}

We now turn our attention to two space dimensions, where the involved Bessel functions do not reduce to standard trigonometric expressions. For $n=2$,

$$
A=k^{2}\left(1-C \ell^{2}\right) /(C-1) \ell^{2}
$$

and

$$
\begin{gathered}
\tilde{B}_{+}(\xi)=\left(1+\frac{a^{2} \xi^{2}}{k^{2}}\right)^{-1}\left[J_{0}(a R)-\frac{a \xi}{k} J_{1}(a R) \frac{K_{0}(k R / \xi)}{K_{1}(k R / \xi)}\right], \\
\tilde{B}_{0}(\xi)=\frac{2 \xi}{k} R \frac{K_{5 / 2}(k R / \xi)}{K_{3 / 2}(k R / \xi)}+1, \\
\tilde{B}_{-}(\xi)=\left(1-\frac{a^{2} \xi^{2}}{k^{2}}\right)^{-1}\left[I_{0}(a R)+\frac{a \xi}{k} I_{1}(a R) \frac{K_{0}(k R / \xi)}{K_{1}(k R / \xi)}\right] .
\end{gathered}
$$

The numerical investigations carried out in [12] led to assertion that flock profiles can only be found when $A>0$. As in the 3D case, we can now give a rigorous theorem and proof, thanks to the explicit computations in Section 2.

Theorem 4.1 Let $W$ be a Quasi-Morse potential in space dimension $n=2$ with parameters within the biologically relevant regime $C>1, \ell<1$. Then flock profiles exist if and only if $A>0$ or equivalently $C \ell^{2}<1$.

We begin by proving a general monotonicity result on the ratio of two modified Bessel functions, which will be used repeatedly throughout the section.

Lemma 4.2 For any $v \geqslant 0$, functions $K_{v+1}(x) /\left(x K_{v}(x)\right), K_{v}(x) /\left(x K_{v+1}(x)\right)$, and $K_{v+1}(x) / K_{v}(x)$ are strictly decreasing functions on $(0, \infty)$. 
Proof Let $w(x)=K_{v+1}(x) /\left(x K_{v}(x)\right)$, which is positive and smooth on $(0, \infty)$. We take the derivative of both sides of $K_{v+1}(x)=x w(x) K_{v}(x)$ and use the recurrence relation

$$
-K_{v}(x)-(v+1) K_{v+1}(x) / x=w(x) K_{v}(x)+x w^{\prime}(x) K_{v}(x)+w(x)\left(v K_{v}(x)-x K_{v+1}(x)\right),
$$

which is equivalent to the differential equation for $w$

$$
2(v+1) w(x)+x w^{\prime}(x)-x^{2} w(x)^{2}+1=0 .
$$

Taking the derivative of (4.3) w.r.t. $x$,

$$
(2 v+3) w^{\prime}(x)+x w^{\prime \prime}(x)-2 x w(x)^{2}-2 x^{2} w(x) w^{\prime}(x)=0 .
$$

We can first get the "boundary conditions" for $w$ near the origin or infinity by asymptotic expansions. When $x$ is close to the origin, one uses (A 6) to deduce

$$
w(x) \sim 2 v x^{-2}, \quad w^{\prime}(x) \sim-4 v x^{-3}<0, \quad w^{\prime \prime}(x) \sim 12 v x^{-4}>0,
$$

for $v>0$ and

$$
w(x) \approx \frac{1}{x^{2}\left(-\frac{1}{2} \ln x-\gamma\right)}, \quad w^{\prime}(x) \sim \frac{4}{x^{3} \ln x}<0, \quad w^{\prime \prime}(x) \sim-\frac{12}{x^{4} \ln x}>0,
$$

for $\mu=0$. When $x$ is large, by the asymptotic expansion (A 7), one gets

$$
w(x) \sim \frac{1}{x}\left(1-\frac{2 v+1}{2 x}\right), \quad w^{\prime}(x) \sim-\frac{1}{x^{2}}<0, \quad w^{\prime \prime}(x) \sim \frac{2}{x^{3}}>0 .
$$

Therefore, $w(x)>0, w^{\prime}(x)<0, w^{\prime \prime}(x)>0$ when $x$ is near origin and $x \rightarrow \infty$. Moreover, $w$ has no local maximum on $(0, \infty)$. Otherwise, if there is a local maximum at $x_{0}$, then $w^{\prime}\left(x_{0}\right)=0, w^{\prime \prime}\left(x_{0}\right) \leqslant 0$. On the other hand, by $(4.4), w^{\prime \prime}\left(x_{0}\right)=2 w\left(x_{0}\right)^{2}>0$, a contradiction.

Next, we show that $w^{\prime}(x)<0$ on $(0, \infty)$. If $w^{\prime}(x)>0$ at some point $x_{1}>0$, then by the fact that $w^{\prime}(x)<0$ when $x$ is large, $w$ must have a local maximum on $\left(x_{1}, \infty\right)$ (because $w$ first increases and then decreases). If $w^{\prime}(x)=0$ at $x_{2}>0$, then by (4.4), $w^{\prime \prime}\left(x_{2}\right)=2 w\left(x_{2}\right)^{2}>0$. Hence, there is a point $\tilde{x}_{2}>x_{2}$, such that $w^{\prime}\left(\tilde{x}_{2}\right)>0$, and it is reduced to the previous case. Therefore, in either situation, there exists a local maximum on $(0, \infty)$, contradicting the statement proved in the last paragraph. This concludes the proof of the strict monotonicity of $w$ on $(0, \infty)$.

Similarly, the monotonicity of $w_{2}(x)=K_{v}(x) /\left(x K_{v+1}(x)\right)$ and $w_{3}(x)=K_{v+1}(x) / K_{v}(x)$ can be proved by using the second-order ODEs,

$$
(2 v-2) w_{2}^{\prime}(x)+2 x^{2} w_{2}(x) w_{2}^{\prime}(x)+2 x w_{2}(x)^{2}-x w_{2}^{\prime \prime}(x)=0
$$

and

$$
2 x^{2} w_{3}(x) w_{3}^{\prime}(x)+(2 v+1) w_{3}(x)-2(v+1) x w_{3}^{\prime}(x)-x^{2} w_{3}^{\prime \prime}(x)=0 .
$$

In all the three cases, the key ingredients of the proof are the right "boundary condition" near the origin and infinity, and $w^{\prime \prime}(x)>0$ at any point $x$ such that $w^{\prime}(x)=0$. 
Lemma 4.2 is needed in the proof of Theorem 4.1, where contrary to the 3D counterpart, the ratios of the Bessel functions do not simplify for even dimensions. The structure of the proof given below would apply in a similar fashion in three dimensions to obtain Theorem 3.1 if the simplified expressions (3.2a)-(3.2c) were omitted. We begin with a discussion of the case $A=0$ for any dimension.

Proof (Theorem 4.1) Suppose $A=0$. Then, in general dimension $n$,

$$
\operatorname{det} M_{0}=\tilde{B}_{0}(\ell)-\tilde{B}_{0}(1)=2 R^{2}\left[\frac{1}{k R / \ell} \frac{K_{\frac{n}{2}+1}(k R / \ell)}{K_{\frac{n}{2}}(k R / \ell)}-\frac{1}{k R} \frac{K_{\frac{n}{2}+1}(k R)}{K_{\frac{n}{2}}(k R)}\right]<0,
$$

as $\ell<1$ and the strict monotonicity of $K_{\frac{n}{2}+1}(x) /\left(x K_{\frac{n}{2}}(x)\right)$ is provided by Lemma 4.2. Hence, no real positive roots of $\operatorname{det} M_{0}$ exist in any dimension. Let us return to the case $n=2$ and suppose $A<0$, then $C \ell^{2}>1$ by (4.1) and $\operatorname{det} M$ can be expressed as

$$
\begin{gathered}
\operatorname{det} M_{-}=\tilde{B}_{-}(\ell)-\tilde{B}_{-}(1)=\left[\left(1-\frac{a^{2} \ell^{2}}{k^{2}}\right)^{-1}-\left(1-\frac{a^{2}}{k^{2}}\right)^{-1}\right] I_{0}(a R) \\
+\frac{a}{k}\left[\ell\left(1-\frac{a^{2} \ell^{2}}{k^{2}}\right)^{-1} \frac{K_{0}(k R / \ell)}{K_{1}(k R / \ell)}-\left(1-\frac{a^{2}}{k^{2}}\right)^{-1} \frac{K_{0}(k R)}{K_{1}(k R)}\right] I_{1}(a R) \\
=\frac{(C-1)\left(1-C \ell^{2}\right)}{C\left(1-\ell^{2}\right)} I_{0}(a R)+\frac{(C-1) a \ell^{2}}{k\left(1-\ell^{2}\right)}\left(\frac{1}{C \ell} \frac{K_{0}(k R / \ell)}{K_{1}(k R / \ell)}-\frac{K_{0}(k R)}{K_{1}(k R)}\right) I_{1}(a R),
\end{gathered}
$$

using (4.2c). The coefficient of $I_{0}(a R)$ is obviously negative. By the monotonicity of $K_{0}(x) /\left(x K_{1}(x)\right)$,

$$
\begin{aligned}
\frac{1}{C \ell} \frac{K_{0}(k R / \ell)}{K_{1}(k R / \ell)}-\frac{K_{0}(k R)}{K_{1}(k R)} & <\ell \frac{K_{0}(k R / \ell)}{K_{1}(k R / \ell)}-\frac{K_{0}(k R)}{K_{1}(k R)} \\
& <k R\left(\frac{\ell}{k R} \frac{K_{0}(k R / \ell)}{K_{1}(k R / \ell)}-\frac{1}{k R} \frac{K_{0}(k R)}{K_{1}(k R)}\right)<0 .
\end{aligned}
$$

This implies that $\operatorname{det} M_{-}<0$. Therefore, there is no flock profile when $A \leqslant 0$.

Next, consider the case $A>0$. The determinant of the coefficient matrix is given as

$$
\operatorname{det} M_{+}=\frac{(C-1)\left(1-C \ell^{2}\right)}{C\left(1-\ell^{2}\right)} J_{0}(a R)-\frac{(C-1) a \ell^{2}}{k\left(1-\ell^{2}\right)}\left[\frac{1}{C \ell} \frac{K_{0}(k R / \ell)}{K_{1}(k R / \ell)}-\frac{K_{0}(k R)}{K_{1}(k R)}\right] J_{1}(a R) .
$$

Let $0=\tilde{R}_{0}<\tilde{R}_{1}<\cdots$ be the simple zeros of $J_{1}(a R)$, then by the relation $J_{0}^{\prime}(x)=J_{1}(x)$, $\tilde{R}_{j}$ are also the critical points of $J_{0}(a R)$. Since $\left.\operatorname{det} M_{+}\right|_{R=\tilde{R}_{j}}$ has alternating signs, det $M_{+}$ has at least one root on $\left(\tilde{R}_{j}, \tilde{R}_{j+1}\right)$, and therefore infinitely many roots on $(0, \infty)$.

Let $R^{*}$ be the first root in the first interval $\left(\tilde{R}_{0}, \tilde{R}_{1}\right)$, then we must have $\left.\tilde{B}_{+}(\ell)\right|_{R=R^{*}}=$ $\left.\tilde{B}_{+}(1)\right|_{R=R^{*}}<0$, as illustrated in Figure 4(a). Otherwise, if $\left.\tilde{B}_{+}(\ell)\right|_{R=R^{*}}=\left.\tilde{B}_{+}(1)\right|_{R=R^{*}} \geqslant 0$, using (4.2a) we deduce

$$
J_{0}\left(a R^{*}\right) \geqslant \frac{a \ell}{k} J_{1}\left(a R^{*}\right) \frac{K_{0}\left(k R^{*} / \ell\right)}{K_{1}\left(k R^{*} / \ell\right)}, \quad J_{0}\left(a R^{*}\right) \geqslant \frac{a}{k} J_{1}\left(a R^{*}\right) \frac{K_{0}\left(k R^{*}\right)}{K_{1}\left(k R^{*}\right)} .
$$




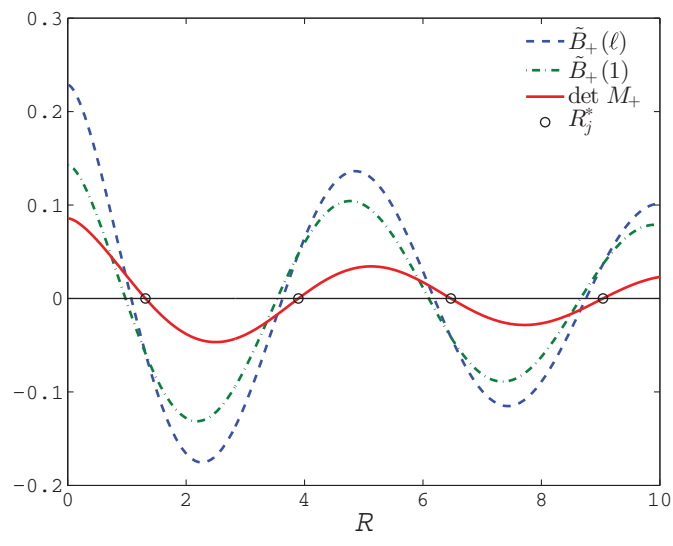

(a) Intersection of $\tilde{B}_{+}(\ell)$ and $\tilde{B}_{+}(1)$ at $R_{j}^{*}$

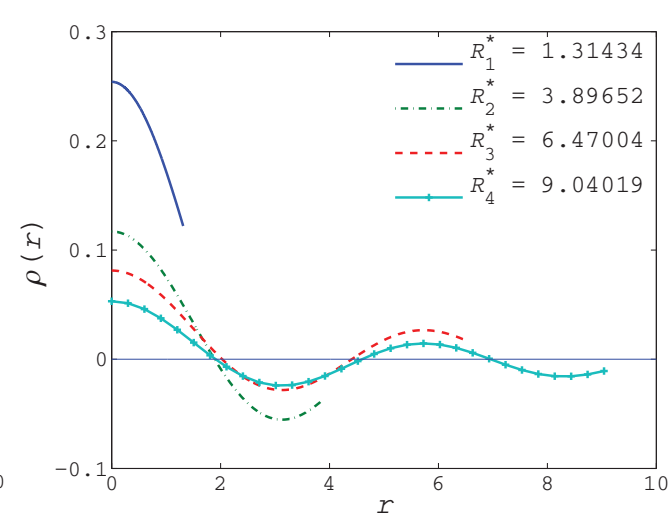

(b) Densities corresponding to $R_{j}^{*}$

FIGURE 4. (Colour online) The roots of determinant $M_{+}$and the corresponding flock profiles. Only the first zero $R_{1}^{*}$ is physically relevant, as the densities become negative on the support $\left(0, R_{k}^{*}\right)$ for the other roots $R_{k}^{*}$. The parameters, $C=10 / 9, \ell=0.75, k=1 / 2$, and $A=1.5$, are the same as in [12].

On the other hand, since $J_{1}\left(a R^{*}\right)$ is positive together with the monotonicity of $K_{0}(x) /\left(x K_{1}(x)\right)$,

$$
J_{0}\left(a R^{*}\right)-\frac{a \ell}{k} J_{1}\left(a R^{*}\right) \frac{K_{0}\left(k R^{*} / \ell\right)}{K_{1}\left(k R^{*} / \ell\right)}>J_{0}\left(a R^{*}\right)-\frac{a}{k} J_{1}\left(a R^{*}\right) \frac{K_{0}\left(k R^{*}\right)}{K_{1}\left(k R^{*}\right)} \geqslant 0,
$$

and consequently

$$
\begin{aligned}
\tilde{B}_{+}(\ell) & =\left(1+\frac{a^{2} \ell^{2}}{k^{2}}\right)^{-1}\left[J_{0}\left(a R^{*}\right)-\frac{a \ell}{k} J_{1}\left(a R^{*}\right) \frac{K_{0}\left(k R^{*} / \ell\right)}{K_{1}\left(k R^{*} / \ell\right)}\right] \\
& >\left(1+\frac{a^{2}}{k^{2}}\right)^{-1}\left[J_{0}\left(a R^{*}\right)-\frac{a}{k} J_{1}\left(a R^{*}\right) \frac{K_{0}\left(k R^{*}\right)}{K_{1}\left(k R^{*}\right)}\right]=\tilde{B}_{+}(1),
\end{aligned}
$$

contradicting the fact that $R^{*}$ satisfies $\left.\operatorname{det} M_{+}\right|_{R=R^{*}}=\left.\tilde{B}_{+}(\ell)\right|_{R=R^{*}}-\left.\tilde{B}_{+}(\ell)\right|_{R=R^{*}}=0$.

Since $\mu_{2}=-\left.\tilde{B}_{+}(1)\right|_{R=R^{*}} \mu_{1}, \mu_{1}$ and $\mu_{2}$ have the same sign. If the corresponding density $\rho(r)=\mu_{1} J_{0}(a r)+\mu_{2}$ at the origin is non-negative, then both $\mu_{1}$ and $\mu_{2}$ are positive. We first factor out $J_{0}\left(a R^{*}\right)$ from equation $\left.\tilde{B}_{+}(\ell)\right|_{R=R^{*}}-\left.\tilde{B}_{+}(\ell)\right|_{R=R^{*}}=0$, i.e.,

$$
J_{0}\left(a R^{*}\right)=\frac{1}{a k\left(1-\ell^{2}\right)}\left[\ell\left(k^{2}+a^{2}\right) \frac{K_{0}\left(k R^{*} / \ell\right)}{K_{1}\left(k R^{*} / \ell\right)}-\left(k^{2}+a^{2} \ell^{2}\right) \frac{K_{0}\left(k R^{*}\right)}{K_{0}\left(k R^{*}\right)}\right] .
$$

Substituting this into $\rho\left(R^{*}\right)=\mu_{1} J_{0}\left(a R^{*}\right)+\mu_{2}=\mu_{1}\left(J_{0}\left(a R^{*}\right)-\left.\tilde{B}_{+}(\ell)\right|_{R=R^{*}}\right)$, we conclude

$$
\rho\left(R^{*}\right)=\frac{a \ell^{2}}{k^{2} R\left(1-\ell^{2}\right)}\left[\frac{k R^{*}}{\ell} \frac{K_{0}\left(k R^{*} / \ell\right)}{K_{1}\left(k R^{*} / \ell\right)}-k R^{*} \frac{K_{0}\left(k R^{*}\right)}{K_{1}\left(k R^{*}\right)}\right] J_{1}\left(a R^{*}\right) \mu_{1}>0 .
$$

Finally, since $R^{*}$ is smaller than the first local minimum $\tilde{R}_{1}$ of $J_{0}(a r), \rho(r)=\mu_{1} J_{0}(a R)+\mu_{2}$ is decreasing on $\left[0, R^{*}\right]$. Thus, the strict positivity of $\rho(r)$ on $\left[0, R^{*}\right]$ results from the strict positivity of $\rho\left(R^{*}\right)$. 
Remark 4.3 Theorem 4.1 lacks the uniqueness result of Theorem 3.1. However, numerical investigations point towards a uniqueness result similar to three dimensions. As an example, we illustrate det $M_{+}$and the densities associated to its roots for a set of parameters investigated in [12] in Figure 4. To prove uniqueness in two dimensions, the possibility of non-negative densities for roots $R^{*}>\tilde{R}_{2}$ and the possibility of multiple solutions $\operatorname{det} M_{+}=0$ in $\left(\tilde{R}_{0}, \tilde{R}_{1}\right)$ have to be ruled out.

\section{Further properties of flock profiles for the Quasi-Morse potential}

Let us remark that there are parameters $(C, \ell)$ such that the convolution equation (1.6) has a solution even though they do not belong to the biologically relevant cases. Flock profiles, as defined in Definition 1.1, can be found by similar proofs as in the previous two sections in the region $\left\{(C, \ell) \mid \ell>1, C \ell^{n-2}>1, C \ell^{n}<1\right\}$, where $U$ has a positive global maximum. These flock profiles are in fact those that have corresponding stable steady solution in the time-reversed first-order swarming system (6.2), and are not observed in simulations, since they are unstable, for both the first-order and the second-order particle models.

The proofs in the previous two sections also indicate the dependence of flock profiles with respect to the size of their support $R^{*}$ parameterized by $\ell$, at least in the asymptotic limit of $\ell$ approaching its lower and upper limits. For example, in $3 \mathrm{D}$, since $R^{*} \in\left(\tilde{R}_{1}, \tilde{R}_{2}\right)$ and $\tilde{R}_{j} \sim O\left(a^{-1}\right)$, we have $R^{*} \sim O\left(a^{-1}\right)$.

In three dimensions, for fixed parameters $C$ and $k$, if $\ell$ is close to its upper limit $C^{-1 / 3}$ in the parameter space, then $a=k \sqrt{\left(1-C \ell^{3}\right) /\left(C \ell^{3}-\ell^{2}\right)}$ is close to zero, and for the auxiliary function $g(R)$ defined in $(3.5 \mathrm{a})$, we have

$$
g(R)=\frac{a}{k} \frac{\left(a^{2} \ell-k^{2}\right) k R+a^{2} \ell(\ell+1)}{a^{2}(\ell+1) k R+k^{2}+a^{2}\left(\ell^{2}+\ell+1\right)} \approx-a R .
$$

The desired root $R^{*}$ can be approximated from the simplified equation $\tan a R-a R=0$, which is simply $R^{*} \approx \bar{r}_{1} \approx 4.49 / a$ in the last step of the proof of Theorem 3.1 . Therefore, as $\ell$ increases to $C^{-1 / 3}$, the radius of support of the flock profile also approaches the first minimum of $r^{-1 / 2} J_{0}(a r)$.

On the other hand, if $\ell$ is close to its lower limit $C^{-1}$, then $a$ diverges, and

$$
g(R) \approx \frac{a}{k} \frac{\ell k R+\ell^{2}+\ell}{(\ell+1) k R+\ell^{2}+\ell+1} .
$$

Since $\ell$ is close to $C^{-1}$ and the desired root $R^{*} \sim a^{-1}$ is close to zero, $g(R)$ can be further simplified to

$$
g(R) \approx \frac{a}{k} \frac{C+1}{C^{2}+C+1}:=a \bar{C},
$$

a constant proportional to $a$. From the asymptotic equation $\tan a R^{*}+a \bar{C}=0$, $a R^{*}$ approaches $\pi / 2$ from above, or $R^{*} \approx \pi /(2 a)$.

Summarizing, in term of the original parameters $k, C$, and $\ell$,

$$
R^{*}=\frac{4.49 \sqrt{1-C^{-2 / 3}}}{k}\left(1-C \ell^{3}\right)^{-1 / 2}+O\left(\left|1-C \ell^{3}\right|\right)
$$



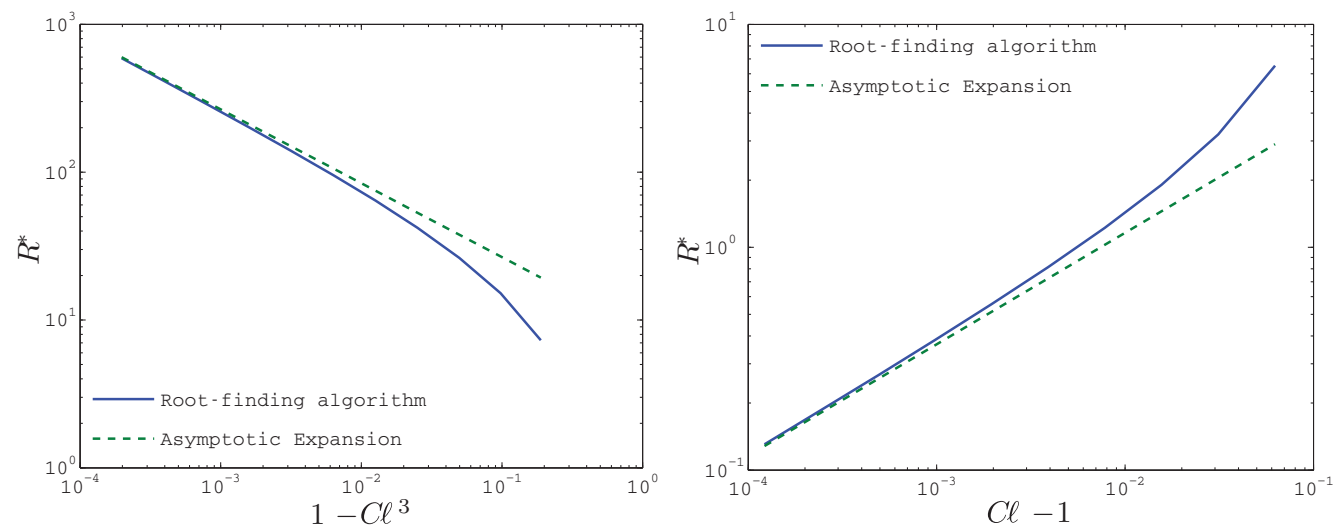

Figure 5. (Colour online) Comparison between the radius of support $R^{*}$ by a root-finding algorithm of $\operatorname{det} M_{+}=0$ and the asymptotic expansion given by (5.1) and (5.2).

when $\ell$ is close to $C^{-1 / 3}$ and

$$
R^{*}=\frac{\pi}{2 k \sqrt{C^{2}-1}}(C \ell-1)^{1 / 2}+O(|C \ell-1|)
$$

when $\ell$ is close to $C^{-1}$. The comparison between these asymptotic expansions of $R^{*}$ with those obtained from solving $\operatorname{det} M_{+}=0$ by a root-finding algorithm is shown in Figure 5. Substituting the above expressions into $M_{+}$, the expansions for $\mu_{1}$ and $\mu_{2}$ can be obtained accordingly.

In two dimensions, the leading-order asymptotic expansion of $R^{*}$ can be derived similarly. When $\ell$ is close to zero, $a \approx k /(\ell \sqrt{C-1})$ is large and $R^{*} \sim a$. Assuming $R^{*}=\ell R_{0}+O\left(\ell^{2}\right)$ for some $R_{0}>0$, then

$$
\left.\tilde{B}_{+}(\ell)\right|_{R=R^{*}} \approx \frac{C-1}{C}\left[J_{0}\left(k R_{0} / \sqrt{C-1}\right)-\frac{1}{\sqrt{C-1}} J_{1}\left(k R_{0} / \sqrt{C-1}\right) \frac{K_{0}\left(k R_{0}\right)}{K_{1}\left(k R_{0}\right)}\right]=O(1)
$$

and

$$
\left.\tilde{B}_{+}(1)\right|_{R=R^{*}} \approx \ell^{2}(C-1)\left[J_{0}\left(k R_{0} / \sqrt{C-1}\right)-\frac{1}{\ell \sqrt{C-1}} J_{1}\left(k R_{0} / \sqrt{C-1}\right) \frac{K_{0}\left(k R_{0} / \ell\right)}{K_{1}\left(k R_{0} / \ell\right)}\right] .
$$

Since $\frac{K_{0}\left(k R_{0} / \ell\right)}{K_{1}\left(k R_{0} / \ell\right)} \rightarrow 1$ as $\ell \rightarrow 0$, we have $\left.\tilde{B}_{+}(1)\right|_{R=R^{*}}=O(\ell)$ and $\left.\left.\tilde{B}_{+}(\ell)\right|_{R=R^{*}} \gg \tilde{B}_{+}(1)\right|_{R=R^{*}}$ unless the leading order in $\left.\tilde{B}_{+}(\ell)\right|_{R=R^{*}}$ vanishes. Therefore, the coefficient $R_{0}$ is determined by

$$
J_{0}\left(k R_{0} / \sqrt{C-1}\right)=\frac{1}{\sqrt{C-1}} J_{1}\left(k R_{0} / \sqrt{C-1}\right) \frac{K_{0}\left(k R_{0}\right)}{K_{1}\left(k R_{0}\right)},
$$

where the positive number $k R_{0} / \sqrt{C-1}$ is smaller than the first positive root of $J_{0}$ since this equation has infinitely many roots. 
When $\ell$ is close to $C^{-1 / 2}, a$ is small and $\operatorname{det} M_{+}$is

$$
\frac{a^{2}\left(1-\ell^{2}\right)}{k^{2}\left(1+a^{2} / k^{2}\right)\left(1+a^{2} \ell^{2} / k^{2}\right)} J_{0}\left(a R^{*}\right)-\frac{a(C-1) \ell^{2}}{k\left(1-\ell^{2}\right)}\left[\frac{1}{C \ell} \frac{K_{0}\left(k R^{*} / \ell\right)}{K_{1}\left(k R^{*} / \ell\right)}-\frac{K_{0}\left(k R^{*}\right)}{K_{1}\left(k R^{*}\right)}\right] J_{1}\left(a R^{*}\right) .
$$

From the fact that $R^{*}$ diverges,

$$
\frac{1}{C \ell} \frac{K_{0}\left(k R^{*} / \ell\right)}{K_{1}\left(k R^{*} / \ell\right)}-\frac{K_{0}\left(k R^{*}\right)}{K_{1}\left(k R^{*}\right)} \rightarrow \frac{1}{C \ell}-1 \approx C^{-1 / 2}-1 \neq 0 .
$$

Therefore, $\operatorname{det} M_{+}=0$ only if $J_{1}\left(a R^{*}\right)$ vanishes to have both terms above of order $a^{2}$. In other words, $R^{*}$ converges to the first positive root of $J_{1}(a r)$. Consequently, the expansions of $R^{*}$ in two dimensions can be obtained.

\section{Variants of Morse-type potentials}

In the previous sections, we have shown that flock profiles precisely exist for the QuasiMorse potential when the parameters $C$ and $\ell$ are in the region $\left\{(C, \ell) \mid C \ell^{n-2}>1, \ell<\right.$ $\left.1, C \ell^{n}<1\right\}$, see Figure 1. The conditions $C \ell^{n-2}>1$ and $\ell<1$ ensure that the potential $U(r)$ is biologically relevant since it has a positive global minimum, while the condition $C \ell^{n}<1$ is related to the non-H-stability of the potential. A similar result for the Morsepotential is presented in [15]. The claim that a positive global minimum of the potential and non-H-stability imply existence of compactly supported flock solutions also seems to be true for other similar potentials of the form $U(r)=V(r)-C V(r / \ell)$, but concentration of density may appear and the dimensionality of the support can vary with $U$. We show some numerical evidence in support of the claim for the generalised Morse-like potential with

$$
V(r)=-e^{-\frac{r p}{p}}, \quad p>0 .
$$

For this potential, the non-H-stability condition $C \ell^{n}<1$ is the same, but the biologically relevant region is given by $\ell<1$ and $C>\ell^{p}$. The numerical simulations were conducted by finding stationary profiles of the first-order swarming system of particles given by

$$
\frac{d x_{i}}{d t}=-\frac{1}{N} \sum_{j \neq i} \nabla W\left(x_{i}-x_{j}\right), \quad i=1, \ldots, N .
$$

Taking these positions and the common velocity $u_{0}$ with $\left|u_{0}\right|^{2}=\alpha / \beta$ as initial data for the second-order system (1.2), the resulting stationary solution is stable [10].

In Figure 6(a), we observe generic, non-concentrated, compactly supported flock profiles for the exponent $p=\frac{1}{2}$ and $\ell<\ell^{*}=C^{1 / p}=0.36$ that appear to converge to a continuous distribution as $N \rightarrow \infty$. The same phenomena are observed for exponents $p \in(0,1)$.

However, this type of aggregation cannot be expected for exponents $p \in(1,2)$. For $C<1$, the density seems to concentrate towards its boundary when $\ell$ approaches $\ell^{*}=C^{1 / p}$, as illustrated in Figure $6(\mathrm{~b})$. For $C>1$, we observe mixed dimensionality of the support in Figure 6(c) for varying exponents $p$ approaching the limit case $p=2$. Flock profiles seem to bifurcate as $p \rightarrow 2$ leading to a concentration on a ring plus a 


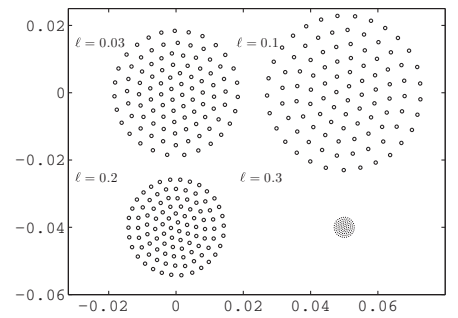

(a) $p=1 / 2, C=0.6$

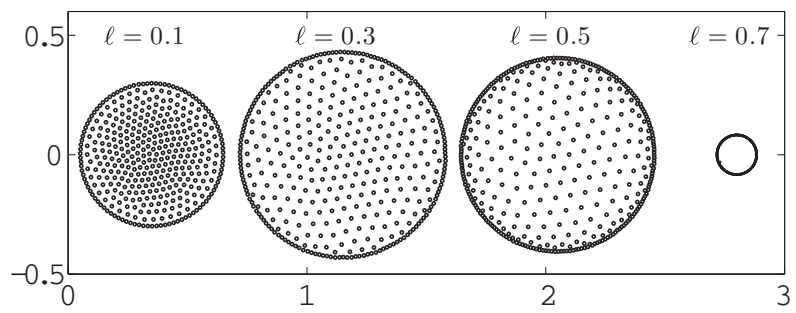

(b) $p=3 / 2, C=0.6, \ell^{*}=C^{1 / p}=0.7114$

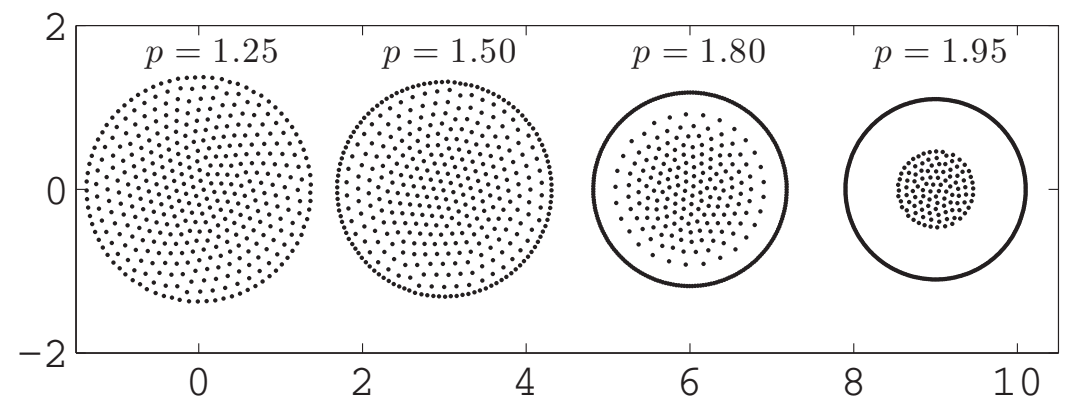

(c) Different $p$ 's with $C=10 / 9, \ell=3 / 4$

FIGURE 6. Flock profiles from the particle simulations of the first-order system (6.2) for the generalised Morse-like potential with $V(r)=-e^{r^{p} / p}$.

continuous distribution inside. To our knowledge, this surprising phenomenon of mixed dimensionality of the support has only been reported in $3 \mathrm{D}$ simulations in $[2,30]$ for purely attractive-repulsive potentials. In a swarming model of locusts in 2D using the Morse potential $[4,5,29]$, the concentration of densities on the (1D) ground can also be reproduced from observations in nature by including additional external gravity force.

This concentration and dimensionality of the support of the steady density is related to the singularity of $U$ near the origin, as has already been demonstrated in [2]. Here we have to argue by numerical experiments as existence proofs will be difficult, partially because of the absence of explicit formulas. Similarly, discussions can be found in [22] for solutions perturbed from a ring solution, and in $[4,5]$ for extensive $1 \mathrm{D}$ examples with $\delta$-concentration on a domain boundary. However, a detailed analytical investigation of these and other properties, such as the integrability of the density near the boundary, remains a challenging question for the potentials considered.

\section{Conclusions}

In this paper, we analyzed the solvability of convolution equations that describe particular solutions in aggregation or self-propelled interacting particle models equipped with radially symmetric interaction potentials. Although models such as (1.2) and (6.2) have been frequently used with various potentials, the analysis of particular solutions, such as flock profiles and rotating mills, is far from complete. We concentrated our attention on the study of flock profiles, defined as compactly supported continuous radial densities 
satisfying equation (1.6). Focusing on the case of the Quasi-Morse potentials introduced in [12], we were able to analytically study the parameter phase portrait of these potentials in two and three dimensions, and proved analytically solvability conditions for the flock profiles that were previously asserted numerically. These findings are summarized in Figure 1: The aggregate potential parameter $A$ determines solvability in the biologically relevant parameter regimes. In three dimensions, we showed the existence and uniqueness of flock profiles for $A>0$, whereas no flock profiles exist if $A \leqslant 0$. The same non-existence result holds true in two dimensions, where flock profiles are shown to exist if and only if $A>0$. The proof of our main Theorems 3.1 and 4.1 is based on a technical discussion of the Bessel functions contained in the definition of the Quasi-Morse potentials and the explicit formulas of their flock profiles obtained in [12]. First, an explicit expression for the convolution $W \star \rho$ was derived for the three cases $A>0, A=0$, and $A<0$. Then a detailed analysis of the resulting expressions enabled us to establish our theorems. A central observation is the fact that the question of existence and uniqueness of flock profiles reduce to the study of roots of a determinant of the coefficient matrix $M$. Due to the simpler functions involved, results obtained in three dimensions are slightly stronger than those obtained in two dimensions.

In summary, this paper is the first, to our knowledge, to complete a full analytical study of the existence of flock profiles in a biologically relevant parameter regime, at least for a particular potential. The analysis of the Quasi-Morse potential and our simulations seem to indicate the existence of flock solutions as long as the potential has a unique positive global minimum and is not $\mathrm{H}$-stable. Characterizing when they are flock profiles is challenging and related to the dimensionality of the support of minimizers of the interaction energy [2]. Proving or disproving these claims for other potentials in (1.2), such as the Morse-type potentials (6.1), as well as the question of stability of such states in the dynamics of the associated partial differential equations (PDEs) however remains an open and challenging problem.

\section{Appendix A Bessel functions and modified Bessel functions}

In this paper, Bessel functions and modified Bessel functions are heavily used to study the analytically more tractable Quasi-Morse type potential (2.1). The definitions and key properties of these Bessel functions, found in standard textbooks of special functions [27], are collected below for readers' convenience.

The Bessel functions of the first kind $J_{v}(x)$ and the second kind $Y_{v}(x)$ are solutions of the equation

$$
x^{2} \frac{d^{2} y}{d x^{2}}+x \frac{d y}{d x}+\left(x^{2}-v^{2}\right) y=0
$$

which are finite and singular at the origin for positive $v$ respectively. The modified Bessel function of the first kind $I_{v}(x)$ and the second kind $K_{v}(x)$ are solutions of the equation

$$
x^{2} \frac{d^{2} y}{d x^{2}}+x \frac{d y}{d x}-\left(x^{2}+v^{2}\right) y=0,
$$

which are exponentially growing and decaying respectively. 
In two and three dimensions considered in this paper, the (modified) Bessel functions with negative order $v$ can be rewritten in terms of those with positive order. In particular, in two dimensions we have

$$
J_{-1}(x)=-J_{1}(x), \quad I_{-1}(x)=I_{1}(x), \quad K_{-1}(x)=K_{1}(x),
$$

and in three dimensions, we have the following explicit representations using the wellknown (hyperbolic) trigonometric functions

$$
\begin{gathered}
J_{1 / 2}(x)=\sqrt{\frac{2}{\pi x}} \sin x, \quad J_{-1 / 2}(x)=\sqrt{\frac{2}{\pi x}} \cos x, \\
K_{1 / 2}(x)=K_{-1 / 2}(x)=\sqrt{\frac{\pi}{2 x}} e^{-x}, \\
I_{1 / 2}(x)=\sqrt{\frac{2}{\pi x}} \sinh x, \quad I_{-1 / 2}(x)=\sqrt{\frac{2}{\pi x}} \cosh x .
\end{gathered}
$$

Recursive relations. In the proof of Lemma 4.2, the following recursive relations for the modified Bessel functions $I_{v}(x)$ and $K_{v}(x)$ are used:

$$
\begin{array}{rr}
I_{v}^{\prime}(x)=I_{v-1}(x)-\frac{v}{x} I_{v}(x), & I_{v}^{\prime}(x)=\frac{v}{x} I_{v}(x)+I_{v+1}(x), \\
K_{v}^{\prime}(x)=-K_{v-1}(x)-\frac{v}{x} K_{v}(x), & K_{v}^{\prime}(x)=\frac{v}{x} K_{v}(x)-K_{v+1}(x) .
\end{array}
$$

In the equivalent integral form, the following are used to evaluate (2.8) and in the proof of Proposition 2.1 in Appendix B:

$$
\int x^{v} I_{v-1}(x) d x=x^{v} I_{v}(x), \quad \int x^{v} K_{v-1}(x) d x=-x^{v} K_{v}(x) .
$$

Asymptotic expansions. In the proof of Lemma 4.2, the following asymptotic expansions of $K_{v}(x)$ for $x>0$ are also needed. When $x>0$ is close to the origin,

$$
K_{v}(x) \approx \begin{cases}-\ln \frac{x}{2}-\gamma, & v=0 \\ \Gamma(v) 2^{v-1} x^{-v}, & v>0\end{cases}
$$

with the Euler constant $\gamma$. When $x$ is large,

$$
K_{v}(x)=\left(\frac{2}{\pi x}\right)^{1 / 2} e^{-x}\left[1+\frac{4 v^{2}-1}{8 x}+\frac{\left(4 v^{2}-1\right)\left(4 v^{2}-9\right)}{2 !(8 x)^{2}}+\cdots\right] \approx K_{1 / 2}(x) .
$$

Additional identities and integrals. The most important identity to simplify the final expressions in (2.8) and in the proof of Proposition 2.1 in Appendix B is

$$
K_{v+1}(x) I_{v}(x)+K_{v}(x) I_{v+1}(x)=\frac{1}{x} .
$$


Finally, we need the following integrals involving products of two Bessel functions [27, p. 87] to evaluate $(2.8)$,

$$
\begin{aligned}
& \int x J_{v}(a x) K_{v}\left(\frac{k x}{\ell}\right) d x=-\frac{\ell^{2}}{k^{2}+a^{2} \ell^{2}}\left[a x J_{v-1}(a x) K_{v}\left(\frac{k x}{\ell}\right)+\frac{k x}{\ell} J_{v}(a x) K_{v-1}\left(\frac{k x}{\ell}\right)\right], \\
& \int x J_{v}(a x) I_{v}\left(\frac{k x}{\ell}\right) d x=\frac{\ell^{2}}{k^{2}+a^{2} \ell^{2}}\left[-a x J_{v-1}(a x) I_{v}\left(\frac{k x}{\ell}\right)+\frac{k x}{\ell} J_{v}(a x) I_{v-1}\left(\frac{k x}{\ell}\right)\right] \\
& \int x I_{v}(a x) K_{v}\left(\frac{k x}{\ell}\right) d x=\frac{\ell^{2}}{a^{2} \ell^{2}-k^{2}}\left[a x I_{v-1}(a x) K_{v}\left(\frac{k x}{\ell}\right)+\frac{k x}{\ell} I_{v}(a x) K_{v-1}\left(\frac{k x}{\ell}\right)\right] \\
& \int x I_{v}(a x) I_{v}\left(\frac{k x}{\ell}\right) d x=\frac{\ell^{2}}{a^{2} \ell^{2}-k^{2}}\left[a x I_{v-1}(a x) I_{v}\left(\frac{k x}{\ell}\right)-\frac{k x}{\ell} I_{v}(a x) I_{v-1}\left(\frac{k x}{\ell}\right)\right]
\end{aligned}
$$

\section{Appendix B Proof of Proposition 2.1}

Here we focus on the integrals related to $V_{\ell}$, because those related to $V$ are obtained by evaluating at $C=1$ and $\ell=1$.

First, we evaluate integral (2.8) when $\rho(s)$ are the linearly independent functions in the general solution (2.3), i.e. constant $1, r^{2}, r^{1-n / 2} J_{n / 2-1}(a r)$ and $r^{1-n / 2} I_{n / 2-1}($ ar $)$ respectively. When $\rho(s)=1$,

$$
\begin{aligned}
& K_{\frac{n}{2}-1}(k r / \ell) \int_{0}^{r} s^{\frac{n}{2}} I_{\frac{n}{2}-1}(k s / \ell) d s+I_{\frac{n}{2}-1}(k r / \ell) \int_{r}^{R} s^{\frac{n}{2}} K_{\frac{n}{2}-1}(k s / \ell) d s \\
& =\left.\frac{\ell}{k} K_{\frac{n}{2}-1}\left(\frac{k r}{\ell}\right) s^{\frac{n}{2}} I_{\frac{n}{2}}\left(\frac{k s}{\ell}\right)\right|_{s=0} ^{r}-\left.\frac{\ell}{k} I_{\frac{n}{2}-1}\left(\frac{k r}{\ell}\right) s^{\frac{n}{2}} K_{\frac{n}{2}}\left(\frac{k s}{\ell}\right)\right|_{s=r} ^{R} \\
& =\frac{\ell}{k} r^{\frac{n}{2}}\left[K_{\frac{n}{2}-1}\left(\frac{k r}{\ell}\right) I_{\frac{n}{2}}\left(\frac{k r}{\ell}\right)+I_{\frac{n}{2}-1}\left(\frac{k r}{\ell}\right) K_{\frac{n}{2}}\left(\frac{k r}{\ell}\right)\right]-\frac{\ell}{k} R^{\frac{n}{2}} I_{\frac{n}{2}-1}\left(\frac{k r}{\ell}\right) K_{\frac{n}{2}}\left(\frac{k R}{\ell}\right) \\
& =\frac{\ell^{2}}{k^{2}} r^{\frac{n}{2}-1}-\frac{\ell}{k} R^{\frac{n}{2}} I_{\frac{n}{2}-1}\left(\frac{k r}{\ell}\right) K_{\frac{n}{2}}\left(\frac{k R}{\ell}\right) .
\end{aligned}
$$

When $\rho(s)=r^{2}$, using (A 5b) and integration by parts, we get

$$
\begin{aligned}
\int s^{\frac{n}{2}+2} K_{\frac{n}{2}-1}(k s / \ell) d s & =-\frac{\ell}{k} s^{\frac{n}{2}+2} K_{\frac{n}{2}}\left(\frac{k s}{\ell}\right)-\frac{2 \ell^{2}}{k^{2}} s^{\frac{n}{2}+1} K_{\frac{n}{2}+1}\left(\frac{k s}{\ell}\right), \\
\int s^{\frac{n}{2}+2} I_{\frac{n}{2}-1}(k s / \ell) d s & =\frac{\ell}{k} s^{\frac{n}{2}+2} I_{\frac{n}{2}}\left(\frac{k s}{\ell}\right)-\frac{2 \ell^{2}}{k^{2}} s^{\frac{n}{2}+1} I_{\frac{n}{2}+1}\left(\frac{k s}{\ell}\right),
\end{aligned}
$$

and hence

$$
\begin{gathered}
K_{\frac{n}{2}-1}(k r / \ell) \int_{0}^{r} s^{\frac{n}{2}+2} I_{\frac{n}{2}-1}(k s / \ell) d s+I_{\frac{n}{2}-1}(k r / \ell) \int_{r}^{R} s^{\frac{n}{2}+2} K_{\frac{n}{2}-1}(k s / \ell) d s \\
=\frac{\ell}{k} r^{\frac{n}{2}+2}\left[K_{\frac{n}{2}-1}\left(\frac{k r}{\ell}\right) I_{\frac{n}{2}}\left(\frac{k r}{\ell}\right)+I_{\frac{n}{2}-1}\left(\frac{k r}{\ell}\right) K_{\frac{n}{2}}\left(\frac{k r}{\ell}\right)\right]
\end{gathered}
$$




$$
\begin{aligned}
+ & \frac{2 \ell^{2}}{k^{2}} r^{\frac{n}{2}+1}\left[I_{\frac{n}{2}-1}\left(\frac{k r}{\ell}\right) K_{\frac{n}{2}+1}\left(\frac{k r}{\ell}\right)-K_{\frac{n}{2}-1}\left(\frac{k r}{\ell}\right) I_{\frac{n}{2}+1}\left(\frac{k r}{\ell}\right)\right] \\
& -\left[\frac{\ell}{k} R^{\frac{n}{2}+2} K_{\frac{n}{2}}\left(\frac{k R}{\ell}\right)+\frac{2 \ell^{2}}{k^{2}} R^{\frac{n}{2}+1} K_{\frac{n}{2}+1}\left(\frac{k R}{\ell}\right)\right] I_{\frac{n}{2}-1}\left(\frac{k r}{\ell}\right) \\
= & \frac{\ell^{2}}{k^{2}} r^{\frac{n}{2}+1}+\frac{2 \ell^{4}}{k^{4}} r^{\frac{n}{2}-1}-R^{\frac{n}{2}+1}\left[\frac{\ell}{k} R K_{\frac{n}{2}}\left(\frac{k R}{\ell}\right)+\frac{2 \ell^{2}}{k^{2}} K_{\frac{n}{2}+1}\left(\frac{k R}{\ell}\right)\right] I_{\frac{n}{2}-1}\left(\frac{k r}{\ell}\right) .
\end{aligned}
$$

Here the terms inside the square bracket of (B1a) or (B 1b) are equal to $\ell / k r$ or $2 n^{2} \ell^{2} /(k r)^{2}$ by the recursive relations (A 5a) and the identity (A 8).

When $\rho(s)=s^{n / 2-1} J_{n / 2-1}(a s)$, using (A 9a) and (A 9b),

$$
\begin{aligned}
& K_{\frac{n}{2}-1}(k r / \ell) \int_{0}^{r} s I_{\frac{n}{2}-1}(k s / \ell) J_{\frac{n}{2}-1}(a s) d s+I_{\frac{n}{2}-1}(k r / \ell) \int_{r}^{R} s K_{\frac{n}{2}-1}(k s / \ell) J_{\frac{n}{2}-1}(a r) d s \\
= & \frac{r k \ell}{a^{2} \ell^{2}+k^{2}}\left[I_{\frac{n}{2}-1}\left(\frac{k r}{\ell}\right) K_{\frac{n}{2}-2}\left(\frac{k r}{\ell}\right)+I_{\frac{n}{2}-2}\left(\frac{k r}{\ell}\right) K_{\frac{n}{2}-1}\left(\frac{k r}{\ell}\right)\right] J_{\frac{n}{2}-1}(a r) \\
& -\frac{R \ell}{a^{2} \ell^{2}+k^{2}}\left[k J_{\frac{n}{2}-1}(a R) K_{\frac{n}{2}-2}\left(\frac{k R}{\ell}\right)+a \ell J_{\frac{n}{2}-2}(a R) K_{\frac{n}{2}-1}\left(\frac{k R}{\ell}\right)\right] I_{\frac{n}{2}-1}\left(\frac{k r}{\ell}\right) \\
= & \frac{\ell^{2}}{a^{2} \ell^{2}+k^{2}} J_{\frac{n}{2}-1}(a r)-\frac{R \ell}{a^{2} \ell^{2}+k^{2}}\left[k J_{\frac{n}{2}-1}(a R) K_{\frac{n}{2}-2}\left(\frac{k R}{\ell}\right)\right. \\
& \left.+a \ell J_{\frac{n}{2}-2}(a R) K_{\frac{n}{2}-1}\left(\frac{k R}{\ell}\right)\right] I_{\frac{n}{2}-1}\left(\frac{k r}{\ell}\right) .
\end{aligned}
$$

Finally, when $\rho(s)=s^{n / 2-1} I_{n / 2-1}($ as), using (A 9c) and (A 9d),

$$
\begin{aligned}
& K_{\frac{n}{2}-1}(k r / \ell) \int_{0}^{r} s I_{\frac{n}{2}-1}(k s / \ell) I_{\frac{n}{2}-1}(a s) d s+I_{\frac{n}{2}-1}(k r / \ell) \int_{r}^{R} s K_{\frac{n}{2}-1}(k s / \ell) I_{\frac{n}{2}-1}(a r) d s \\
= & -\frac{r k \ell}{a^{2} \ell^{2}-k^{2}}\left[I_{\frac{n}{2}-1}\left(\frac{k r}{\ell}\right) K_{\frac{n}{2}-2}\left(\frac{k r}{\ell}\right)+I_{\frac{n}{2}-2}\left(\frac{k r}{\ell}\right) K_{\frac{n}{2}-1}\left(\frac{k r}{\ell}\right)\right] I_{\frac{n}{2}-1}(a r) \\
& +\frac{R \ell}{a^{2} \ell^{2}-k^{2}}\left[k I_{\frac{n}{2}-1}(a R) K_{\frac{n}{2}-2}\left(\frac{k R}{\ell}\right)+a \ell I_{\frac{n}{2}-2}(a R) K_{\frac{n}{2}-1}\left(\frac{k R}{\ell}\right)\right] \\
= & -\frac{\ell^{2}}{a^{2} \ell^{2}+k^{2}} J_{\frac{n}{2}-1}(a r)+\frac{R \ell}{a^{2} \ell^{2}-k^{2}}\left[k I_{\frac{n}{2}-1}(a R) K_{\frac{n}{2}-2}\left(\frac{k R}{\ell}\right)\right. \\
& \left.+a \ell I_{\frac{n}{2}-2}(a R) K_{\frac{n}{2}-1}\left(\frac{k R}{\ell}\right)\right] I_{\frac{n}{2}-1}\left(\frac{k r}{\ell}\right) .
\end{aligned}
$$

Putting all the integrals together, we conclude the explicit form (2.9) for the convolution $W \star \rho$. For example, when $A>0, \rho(r)=\mu_{1} r^{1-\frac{n}{2}} J_{\frac{n}{2}-1}(a r)+\mu_{2}$, collecting the terms in the integral (2.7), we get

$$
\begin{aligned}
(W \star \rho)(r)= & \mu_{2} \frac{C \ell^{n}-1}{k^{2}}+\mu_{1} r^{1-\frac{n}{2}}\left(\frac{C \ell^{n}}{a^{2} \ell^{2}+k^{2}}-\frac{1}{a^{2}+k^{2}}\right) J_{\frac{n}{2}-1}(a r) \\
& -r^{1-\frac{n}{2}}\left\{\mu_{1} \frac{C \ell^{n-1} R}{a^{2} \ell^{2}+k^{2}}\left[k J_{\frac{n}{2}-1}(a R) K_{\frac{n}{2}-2}\left(\frac{k R}{\ell}\right)+a \ell J_{\frac{n}{2}-2}(a R) K_{\frac{n}{2}-1}\left(\frac{k R}{\ell}\right)\right]\right.
\end{aligned}
$$




$$
\begin{aligned}
& \left.+\mu_{2} \frac{\ell}{k} K_{\frac{n}{2}}\left(\frac{k R}{\ell}\right)\right\} I_{\frac{n}{2}-1}\left(\frac{k r}{\ell}\right)+r^{1-\frac{n}{2}}\left\{\mu _ { 1 } \frac { R } { a ^ { 2 } + k ^ { 2 } } \left[k J_{\frac{n}{2}-1}(a R) K_{\frac{n}{2}-2}(k R)\right.\right. \\
& \left.\left.+a J_{\frac{n}{2}-2}(a R) K_{\frac{n}{2}-1}(k R)+\mu_{2} \frac{1}{k} K_{\frac{n}{2}}(k R)\right]\right\} I_{\frac{n}{2}-1}(k r) .
\end{aligned}
$$

The first term $\mu_{2}\left(C \ell^{n}-1\right) / k^{2}$ is the desired constant $D$, and the factor $C \ell^{n} /\left(a^{2} \ell^{2}+k^{2}\right)-$ $1 /\left(a^{2}+k^{2}\right)$ in the second term vanishes by the definition of $a^{2}$. The rest of the terms are a linear combination of $I_{\frac{n}{2}-1}(k r / \ell)$ and $I_{\frac{n}{2}-1}(k r)$, and they can be rearranged into the form (2.3) with the coefficient of $\mu_{2}$ normalized to one to simplify the later proofs. The explicit form for $W \star \rho$ when $A=0$ or $A<0$ has similar structures, and its simplification leads to the final expression (2.9).

\section{Acknowledgement}

Jose Carrillo was supported by projects MTM2011-27739-C04-02 and 2009-SGR-345 from Agència de Gestió d'Ajuts Universitaris i de Recerca-Generalitat de Catalunya. Jose Carrillo acknowledges support from the Royal Society through the Wolfson Research Merit Award. Jose Carrillo, Yanghong Huang, and Stephan Martin were supported by Engineering and Physical Sciences Research Council grant number EP/K008404/1.

\section{References}

[1] Albi, G., Balagué, D., Carrillo, J. A. \& VonBrecht, J. (to appear) Stability analysis of flock and mill rings for 2 nd order models in swarming. SIAM J. Appl. Math..

[2] Balagué, D., Carrillo, J. A., Laurent, T. \& Raoul, G. (2013) Dimensionality of local minimizers of the interaction energy. Arch. Rat. Mech. Anal. 209(3), 1055-1088.

[3] Balagué, D., Carrillo, J. A., Laurent, T. \& Raoul, G. (2013) Nonlocal interactions by repulsive-attractive potentials: Radial ins/stability. Phys. D 260, 5-25.

[4] Bernoff, A. J. \& Topaz, C. M. (2011) A primer of swarm equilibria. SIAM J. Appl. Dyn. Syst. 10(1), 212-250.

[5] Bernoff, A. J. \& Topaz, C. M. (2013) Nonlocal aggregation models: A primer of swarm equilibria. SIAM Rev. 55(4), 709-747.

[6] Bertozzi, A. L., Carrillo, J. A. \& Laurent, T. (2009) Blow-up in multidimensional aggregation equations with mildly singular interaction kernels. Nonlinearity 22(3), 683-710.

[7] Bertozzi, A. L., von Brecht, J. H., Sun, H., Kolokolnikov, T. \& Uminsky, D. (to appear) Ring patterns and their bifurcations in a nonlocal model of biological swarms. Comm. Math. Sci.

[8] Camazine, S., Deneubourg, J.-L., Franks, N. R., Sneyd, J., Theraulaz, G. \& Bonabeau, E. (2003) Self-Organization in Biological Systems. Princeton Studies in Complexity, Princeton University Press, Princeton, NJ (reprint of the 2001 original).

[9] Carrillo, J. A., D’Orsogna, M. R. \& Panferov, V. (2009) Double milling in self-propelled swarms from kinetic theory. Kinet. Relat. Models 2(2), 363-378.

[10] Carrillo, J. A., Huang, Y. \& Martin, S. (2014) Nonlinear stability of flock solutions in second-order swarming models. Nonlinear Anal. Real World Appl. 17, 332-343.

[11] Carrillo, J. A., Klar, A., Martin, S. \& Tiwari, S. (2010) Self-propelled interacting particle systems with roosting force. Math. Mod. Meth. Appl. Sci. 20, 1533-1552.

[12] Carrillo, J. A., Martin, S. \& Panferov, V. (2013) A new interaction potential for swarming models. Phys. D 260, 112-126. 
[13] Chuang, Y.-L., D’Orsogna, M. R., Marthaler, D., Bertozzi, A. L. \& Chayes, L. S. (2007) State transitions and the continuum limit for a $2 \mathrm{D}$ interacting, self-propelled particle system. Phys. D 232(1), 33-47.

[14] Couzin, I. D. \& Krause, J. (2003) Self-organization and collective behavior of vertebrates. Adv. Study Behav. 32, 1-67.

[15] D’Orsogna, M. R., Chuang, Y.-L., Bertozzi, A. L. \& Chayes, L. S. (2006) Self-propelled particles with soft-core interactions: Patterns, stability, and collapse. Phys. Rev. Lett. 96(10), 104302.

[16] Fetecau, R. C. \& Huang, Y. (2013) Equilibria of biological aggregations with nonlocal repulsive-attractive interactions. Phys. D 260, 49-64.

[17] Fetecau, R. C., Huang, Y. \& Kolokolnikov, T. (2011) Swarm dynamics and equilibria for a nonlocal aggregation model. Nonlinearity 24(10), 2681-2716.

[18] Hildenbrandt, H., Carere, C. \& Hemelrijk, C. K. (2010) Self-organised complex aerial displays of thousands of starlings: A model. Behav. Ecology 107(21), 1349-1359.

[19] Holm, D. D. \& Putkaradze, V. (2005) Aggregation of finite-size particles with variable mobility. Phys. Rev. Lett. 95, 226106.

[20] Holm, D. D. \& PutKaradze, V. (2006) Formation of clumps and patches in self-aggregation of finite-size particles. Phys. D 220(2), 183-196.

[21] Huth, A. \& Wissel, C. (1992) The simulation of the movement of fish schools. J. Theor. Biol. 156, 365-385.

[22] Kolokolnikov, T., Huang, Y. \& Pavlovski, M. (2013) Singular patterns for an aggregation model with a confining potential. Phys. D 260, 65-76.

[23] Kolokonikov, T., Sun, H., Uminsky, D. \& Bertozzi, A. (2011) Stability of ring patterns arising from 2d particle interactions. Phys. Rev. E 84(1), 015203.

[24] Levine, H., Rappel, W.-J. \& Cohen, I. (2000, December) Self-organization in systems of self-propelled particles. Phys. Rev. E 63, 017101.

[25] Lieb, E. H. \& Loss, M. (2001) Analysis, 2nd ed., Graduate Studies in Mathematics, Vol. 14, American Mathematical Society, Providence, RI.

[26] Lukeman, R., Li, Y. X. \& Edelstein-Keshet, L. (2010) Inferring individual rules from collective behavior. Proc. Natl. Acad. Sci. USA 107(28), 12576-12580.

[27] Magnus, W., Oberhettinger, F. \& Soni, R. P. (1966) Formulas and Theorems for the Special Functions of Mathematical Physics, Springer-Verlag, New York, NY.

[28] Mogilner, A. \& Edelstein-Keshet, L. (1999) A non-local model for a swarm. J. Math. Biol. 38, 534-570.

[29] Topaz, C. M., Bernoff, A. J., Logan, S. \& Toolson, W. (2008) A model for rolling swarms of locusts. Eur. Phys. J. Spec. Top. 157(1), 93-109.

[30] von Brecht, J. H. \& UMinsky, D. (2012) On soccer balls and linearized inverse statistical mechanics. J. Nonlinear Sci. 22(6), 935-959.

[31] von Brecht, J. H., Uminsky, D., Kolokolnikov, T. \& Bertozzi, A. L. (2012) Predicting pattern formation in particle interactions. Math. Models Methods Appl. Sci. 22, 1140002, 31. 